\title{
Recommendations for Assessing Cognitive Risks in Young Children Treated for Ependymoma for Clinical and Research Protocols: Evidence from a Systematic Literature Review
}

\author{
Matthew C.H.J. Morrall ${ }^{*}, 1$, Nicola J. Pitchford ${ }^{2,3}$, Emma C. Waters ${ }^{1}$, Kate L. Ablett ${ }^{1}$, \\ Helen Stocks ${ }^{1}$, David Walker ${ }^{3}$ and Richard G. Grundy ${ }^{3}$ \\ ${ }^{1}$ Paediatric Neuropsychology, The Leeds Teaching Hospitals NHS Trust, Leeds, LS1 3EX (MM, EW, KA, \\ HS), United Kingdom \\ ${ }^{2}$ School of Psychology, University of Nottingham, Nottingham, NG7 2RD (NP), United Kingdom \\ ${ }^{3}$ Children's Brain Tumour Research Centre, Academic Division of Child Health, University of Nottingham, \\ Queen's Medical Centre, Nottingham, NG7 2RD (DW, RG), United Kingdom
}

\begin{abstract}
Background: Current treatment approaches for pediatric ependymoma differ between North American and European studies. Post-surgical adjuvant irradiation is used in children aged $<36$ months in North America, whilst European approaches use chemotherapy to avoid or defer radiotherapy until three years of age, in order to avoid late neurocognitive toxicity. To establish evidence for the effects of cranial radiotherapy in children aged $<36$ months with ependymoma on neurocognitive outcomes, we conducted a systematic literature review assessing methodological approaches for measuring neurocognitive outcome. Methods: Eight databases were selected to perform an advanced search, retrieval and systematic review of papers describing neurocognitive outcome in children diagnosed with ependymoma who received cranial radiotherapy at $<36$ months. Results: Limitations of published data permitted descriptive analysis only. Considerable variation in reporting survival rates, techniques and timing of psychometric testing and the results of neurocognitive outcomes was identified. Conclusions: The review identified significant inconsistencies of neurocognitive testing, particularly literacy skills, developmental time points for testing and methods of data reporting. The role of the cerebellum for cognitive development, especially reading, has been inadequately evaluated in published studies. Recommendations are made to improve assessment methods, and time points for testing, so that reports do not fail to identify children who acquire deficits as they mature through childhood and adolescence. We conclude that claims that radiation treatment for ependymoma administered aged $<36$ months is associated with limited neurocognitive consequences, are not supported by the literature.
\end{abstract}

Keywords: Paediatric, ependymoma, cognitive, risk, outcome.

\section{INTRODUCTION}

Ependymoma arising at less than 16 years of age account for $10 \%$ of brain tumours in the age group, $>50 \%$ present in the pre-school age group $(<5 \mathrm{yrs})$ and $<80 \%$ presenting by eight years of age $[1,2]$. Ninety percent of pediatric ependymomas are intracranial in origin with two-thirds arising from the lining of the fourth ventricle in the posterior fossa [3]. The young age bias coupled with the complexities of achieving complete resection of tumour involving the brain stem and cerebellum have contributed to poor outcomes because of incomplete resections and restricted use of radiotherapy linked to risks of neurotoxicity affecting cognitive development and other long-term clinical sequelae [4-6].

Concerns regarding the long-term cognitive and learning impairments of irradiating immature brain structures, particularly supratentorial regions and its

*Address correspondence to this author at the Paediatric Neuropsychology, E Floor, Martin Wing, Leeds General Infirmary, Great George Street, Leeds LS1 3EX, United Kingdom; Tel: 01132065897 ; Fax: 0113 3923736;

E-mail: matthew.morrall@leedsth.nhs.uk impact on developing cognitive functions, have led some centres to employ strategies to delay or avoid the delivery of radiotherapy by using chemotherapy first. Understandably, much research in neurooncology focuses on survival rates as primary outcome measures, whilst lower priorities have historically been allocated to neurocognitive and learning outcome measures as drivers for change in treatments [7]. An exception to this [8] is the reporting from North America of the use of highly conformal radiotherapy as the primary adjuvant therapy in children aged $<36$ months with ependymoma [9]. This approach contrasts with many European centres which are continuing to use radiotherapy-deferral strategies with adjuvant chemotherapy.

This difference in clinical practice highlights the importance of considering the neurocognitive consequences for radiotherapy given to the very immature brain, particularly the posterior fossa [10]. Although the cerebellum has been thought to be devoted almost entirely to motor control [11], namely skilled voluntary movements, muscle tone, posture and gait, a growing body of empirical data implicates the developing 
cerebellum in diverse higher cognitive functions [12], especially acquisition of literacy skills [13-15]. Furthermore, neuroendocrine sequelae and second cancers after radiotherapy, adversely influence quality of survival [16-18]. In order to investigate the impact of different treatment regimes [19] a systematic literature review of publications describing the neurocognitive outcomes of children with ependymoma who received radiotherapy at $<36$ months of age was conducted.

\section{MATERIALS AND METHODS}

\section{Search Strategy}

An advanced search was performed in AMED, BIOSIS Previews, CAB Abstracts, EMBASE, Ovid MEDLINE, PsycINFO, CINAHL and Cochrane Library for articles published in English from database commencement to date. All databases were searched using the terms: ((ependymoma*) OR (post ${ }^{*}$ adj2

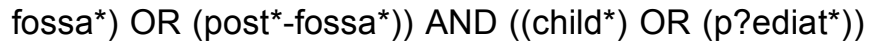
AND ((radiotherapy*) OR (radiat* adj2 therap*) OR (irradiat*) OR (stereotactic adj2 surger*) OR (gamma adj2 knife) OR (IMRT) OR (chemotherap* adj2 wafer*) OR (proton adj2 therap*) OR (photon adj2 therap*) OR (brachytherap*)) AND ((neurocognit*) OR (neuro adj2 cognit $^{\star}$ ) OR (psychometric*) OR (neurometric*) OR (learning* $^{*}$ OR (educat*) OR (neuropsych*) OR $($ psycholog*) OR (cognit*)).

\section{Selection Criteria}

Three members of the review team read the retrieved papers independently and identified data for the agreed categories presented in Tables 1, 2 and 3. Inclusion was dependent on two criteria:

1. The paper reported participants receiving irradiation at three years of age or under for the treatment of ependymoma.

2. The paper reported participants' neurocognitive or psychometric outcomes.

Level of evidence was determined independently by three investigators using indicators as defined by the Centre for Evidence Based Medicine [20] (Table 1).

\section{Statistical Analysis}

The retrieved data did not permit meta-analysis or use of a vote count procedure because of inconsistencies across studies in their use of comparable neuropsychological and psychometric assessments or lack of detailed reporting of children with significantly impaired performance. Consequently, a descriptive analysis was performed. Data were presented using the following categories: number of patients with ependymoma; age at irradiation; grade and site; nonradiological treatments received; residual disease stated; presence of hydrocephalus; radiation dose; survival rate; psychometry used; described impairment; global outcomes and level of evidence [20].

Table 1: Oxford Centre for Evidence-Based Medicine Levels of Evidence Summary

\begin{tabular}{|c|c|}
\hline $\begin{array}{c}\text { Levels of } \\
\text { Evidence }\end{array}$ & Requirement \\
\hline \hline $1 \mathrm{a}$ & Systematic review (with homogeneity) of RCTs \\
\hline $1 \mathrm{~b}$ & $\begin{array}{c}\text { Individual RCT (with narrow confidence } \\
\text { interval) }\end{array}$ \\
\hline $1 \mathrm{c}$ & All or none case series \\
\hline $2 \mathrm{a}$ & $\begin{array}{c}\text { Systematic review (with homogeneity) of cohort } \\
\text { studies }\end{array}$ \\
\hline $2 \mathrm{~b}$ & Individual cohort study \\
\hline $2 \mathrm{c}$ & 'Outcomes' research \\
\hline $3 \mathrm{a}$ & $\begin{array}{c}\text { Systematic review (with homogeneity) of case } \\
\text { control studies }\end{array}$ \\
\hline $3 \mathrm{~b}$ & Individual case-control study \\
\hline 4 & Case series \\
\hline 5 & Expert opinion without critical appraisal \\
\hline
\end{tabular}

\section{RESULTS}

In total, 291 papers were retrieved. Figure 1 illustrates the retrieval process which was completed in accordance with the Preferred Reporting Items for Systematic Reviews and Meta-Analyses (PRISMA) statement [21].

After removing duplicates, the remaining 141 papers were evaluated to determine inclusion. Nine studies met the inclusion criteria (Table 2). A further five studies did not provide specific information for age at the time of irradiation but stated that patients were less than five years (Table 3 ). An additional 11 studies indicated the inclusion of patients with ependymoma but age could not be determined from data provided (Supplementary Table).

\section{Retrieved Studies of Children $<36$ Months Diagnosed with Ependymoma}

Nine references were retrieved from 1990-2011 (Table 1). Of the retrieved references, $88.8 \%(8 / 9$ papers) met level $2 c$ [20] for quality of evidence with 


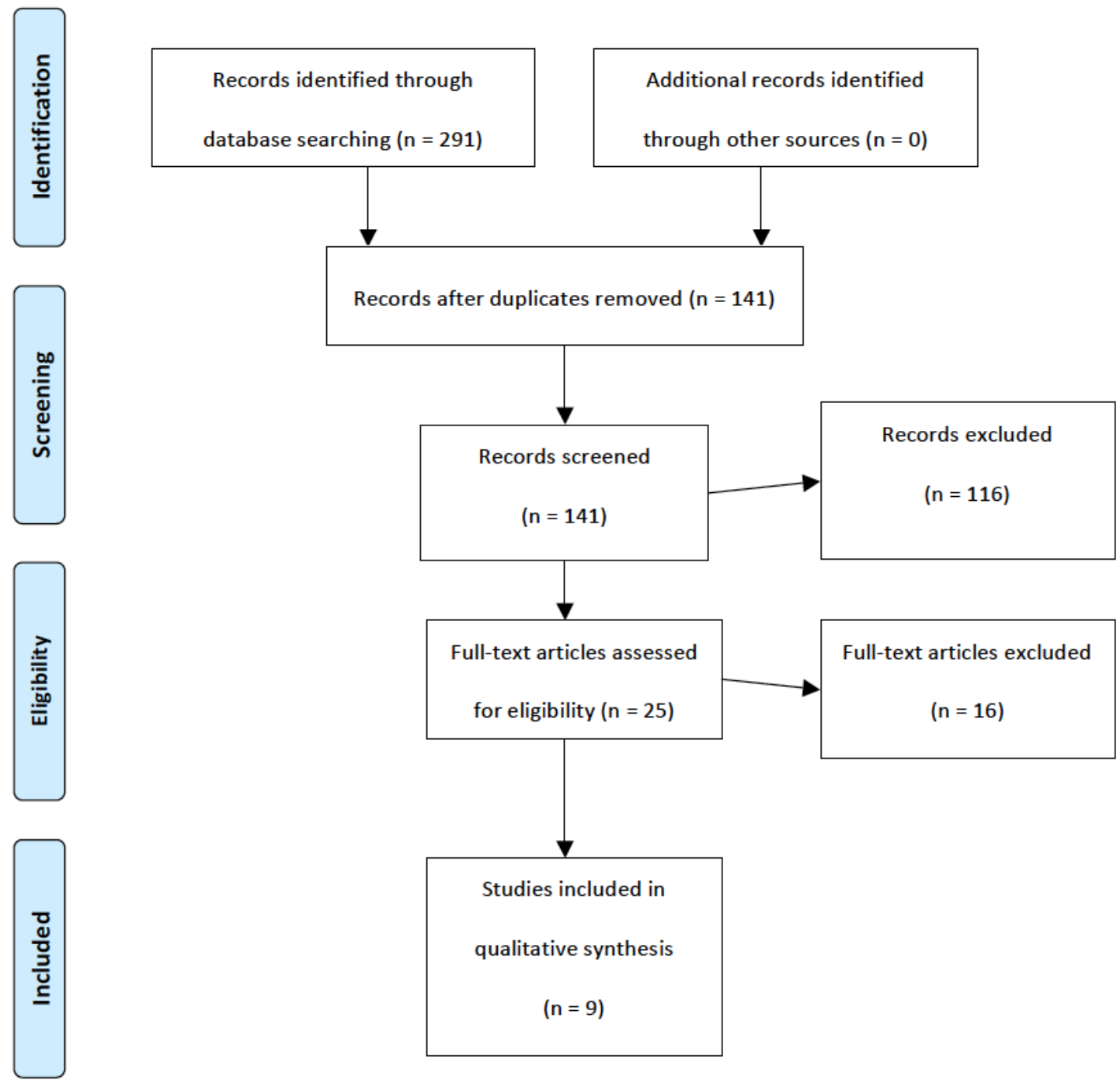

Figure 1: Retrieval Algorithm in accordance with PRISMA Guidelines.

[25] at $3 b$ [20]. Two pairs of papers described the same patients $[8,22,24,27]$. The total number of patients involved in all nine studies was 184 . Of these, $35.9 \%$ $(66 / 184)$ were irradiated at $<36$ months $(0.67$ [8 months]-2 years). The first study [7] contained two protocols for irradiation where the highest dose was 70.4Gy prior to 2001 and then 59.4Gy from 2001 onwards. Mean and standard deviation for all ages were not calculated as three papers $[8,26,27]$ did not specify a mean but stated patients were irradiated at $<36$ months. Of the $35.9 \%$ irradiated at $<36$ months, $80.3 \%(53 / 66)$ had an infratentorial location with $13.6 \%$ $(9 / 66)$ having supratentorial. The remaining four patients $(6.5 \%)$ irradiated at $<36$ months from one paper [23] were not identified as either infra- or supratentorial.

Of the 66 patients, all received neurosurgery. For $13.6 \%(9 / 66)$ the level of resection was unspecified,
$86.4 \%(57 / 66)$ had Gross-Total Resection (GTR), 6.1\% (4/66) had Near-Total Resection (NTR) and $9.1 \%$ $(6 / 66)$ had Subtotal Resection (STR). Of all patients, $25.8 \%(17 / 66)$ received chemotherapy in addition to irradiation. A maximum of seven patients may have received chemotherapy in addition to irradiation but this is not described [7, 25]. Where reported, hydrocephalus was present in $74.2 \%(49 / 66)$ of patients irradiated at $<36$ months.

\section{Radiation and Chemotherapy Treatment Received}

Radiation dosage was reported in $77.7 \%$ (7/9) of the studies, ranging from 40-70.4Gy. For [7] in the 19942001 period, patients with complete tumour excision received hyperfractionated $\mathrm{RT}$ (1.1. Gy twice a day) to the tumour bed plus $1-2 \mathrm{~cm}$ margins up to a total dose of $70.4 \mathrm{~Gy}$. Where residual tumour was identified, four chemotherapy (CT) doses with vincristine, etoposide 


\begin{tabular}{|c|c|c|c|}
\hline \multicolumn{2}{|c|}{ 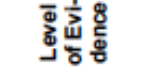 } & \& & $\stackrel{8}{2}$ \\
\hline \multicolumn{2}{|c|}{ 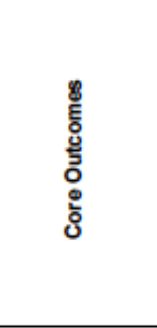 } & 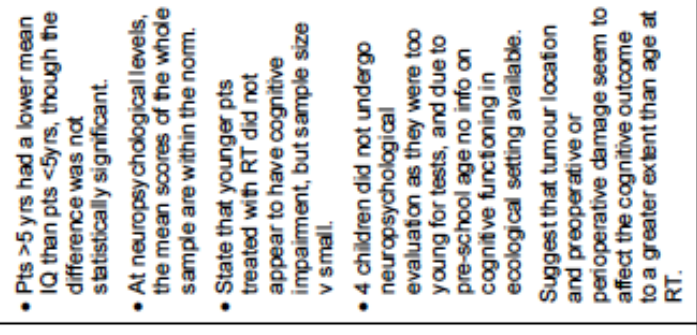 & 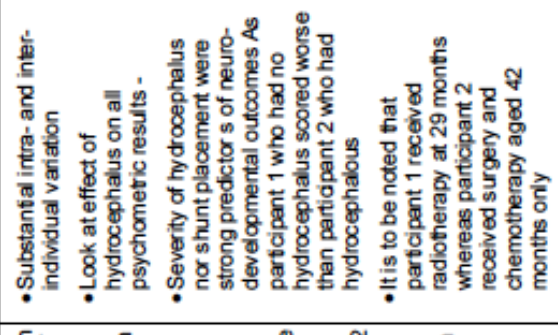 \\
\hline \multicolumn{2}{|c|}{ 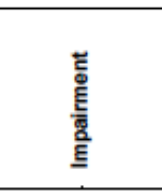 } & 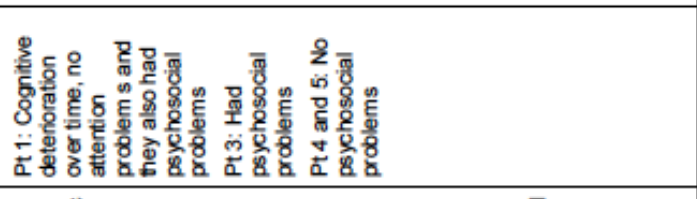 & 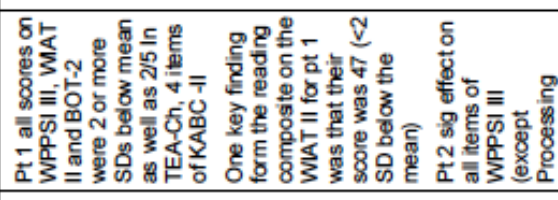 \\
\hline \multicolumn{2}{|c|}{ 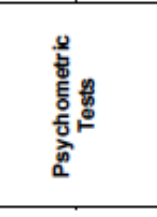 } & 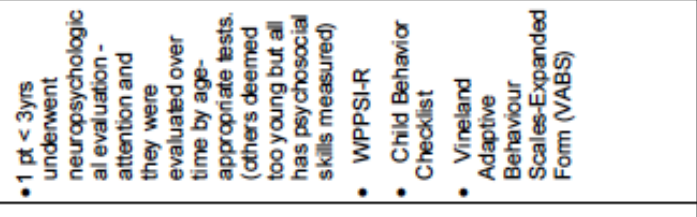 & 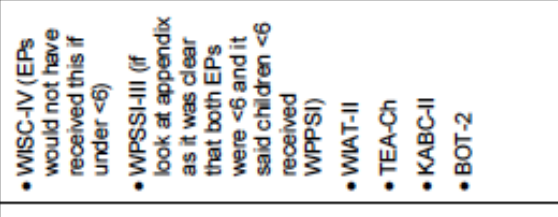 \\
\hline \multicolumn{2}{|c|}{ 惡。 } & 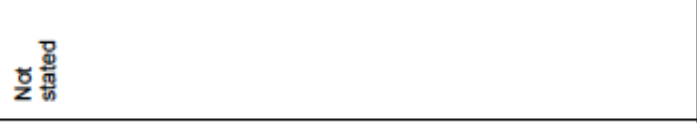 & 兽 \\
\hline \multicolumn{2}{|c|}{ 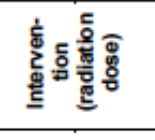 } & 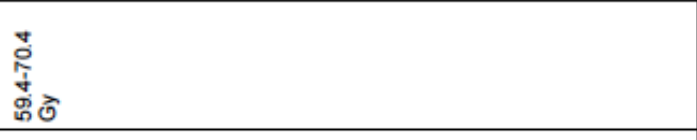 & 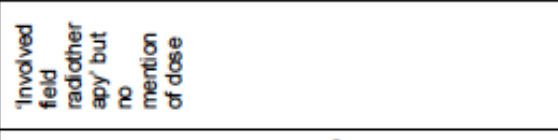 \\
\hline \multicolumn{2}{|c|}{ 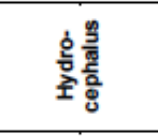 } & 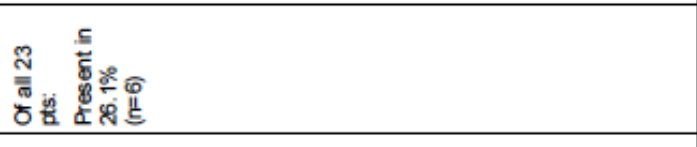 & 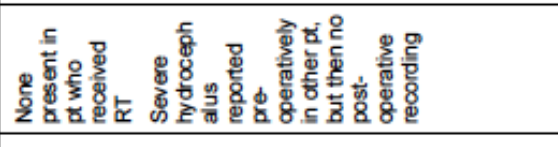 \\
\hline \multicolumn{2}{|c|}{ 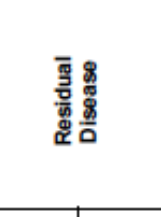 } & 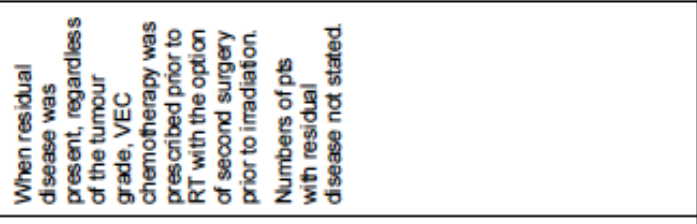 & 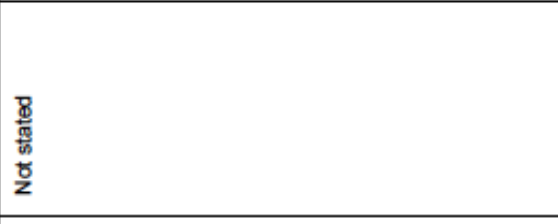 \\
\hline \multirow{2}{*}{ 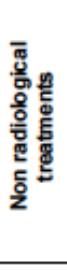 } & 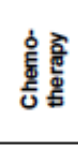 & 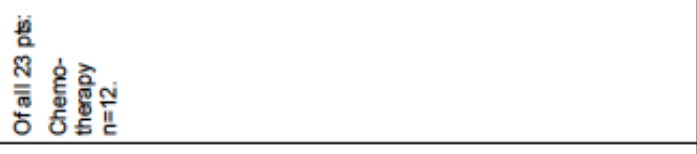 & 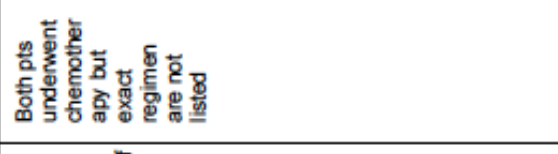 \\
\hline & 密 & 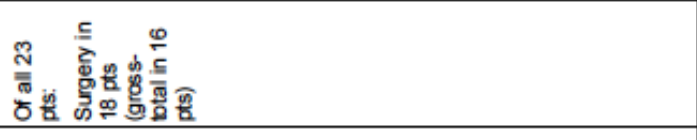 & 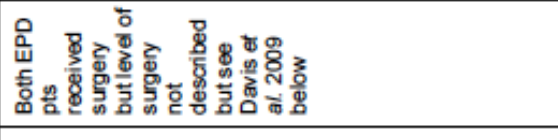 \\
\hline \multicolumn{2}{|c|}{ 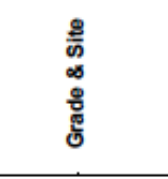 } & 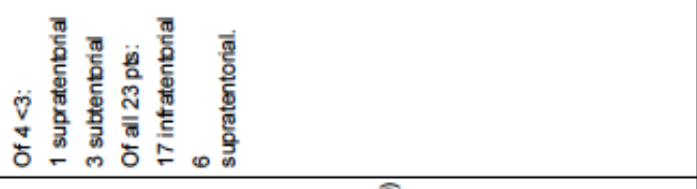 & 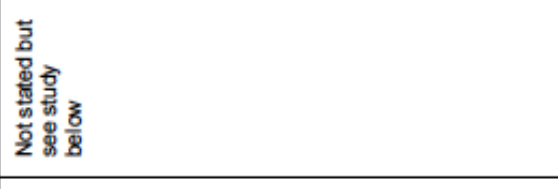 \\
\hline \multicolumn{2}{|c|}{ 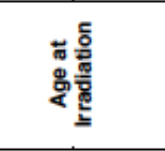 } & 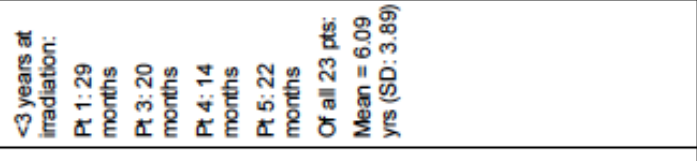 & 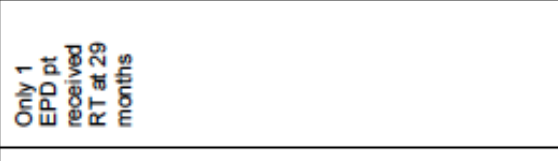 \\
\hline \multicolumn{2}{|c|}{ 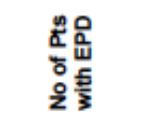 } & 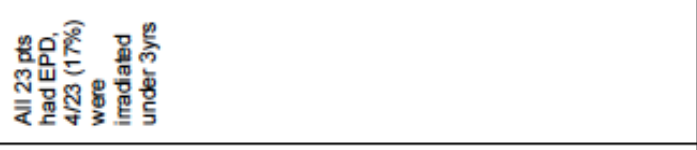 & 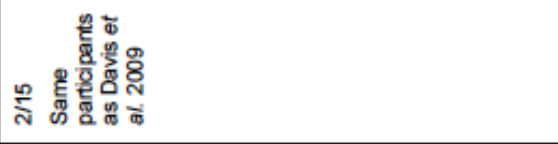 \\
\hline \multicolumn{2}{|c|}{$\frac{\frac{p}{\phi}}{\Phi}$} & 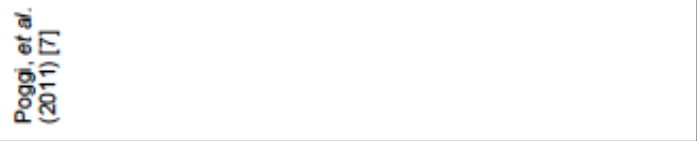 & 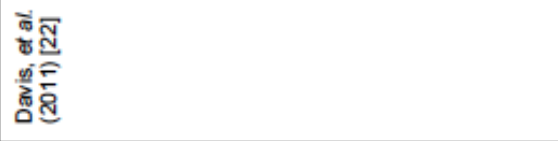 \\
\hline
\end{tabular}




\begin{tabular}{|c|c|c|}
\hline 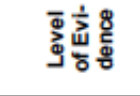 & & $\stackrel{2}{0}$ \\
\hline 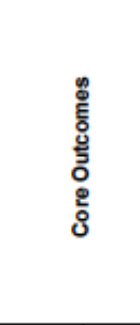 & 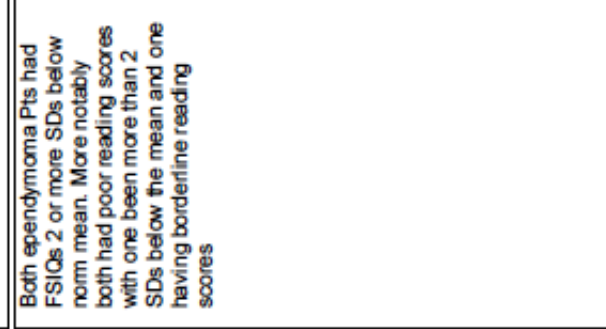 & 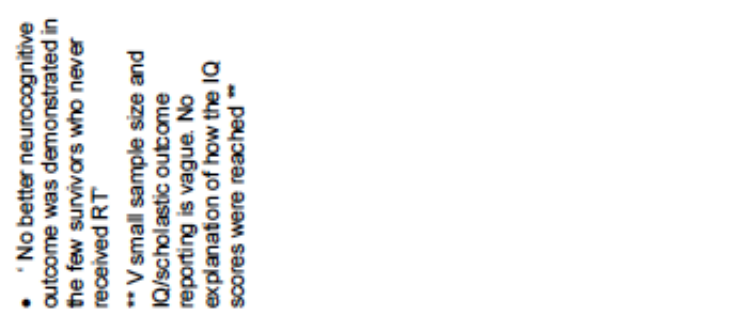 \\
\hline 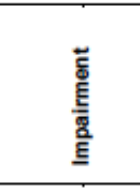 & 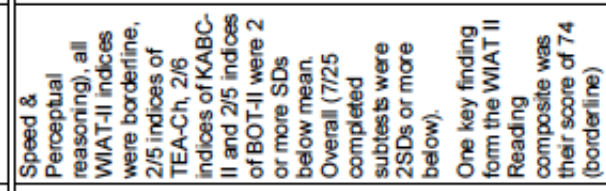 & 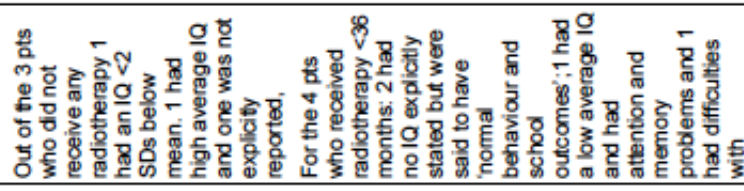 \\
\hline 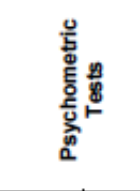 & & 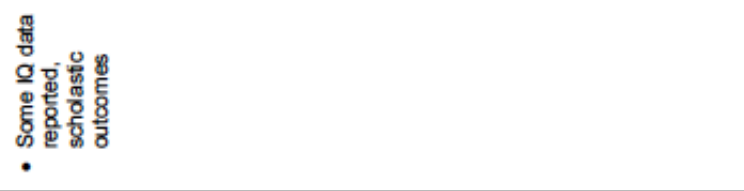 \\
\hline 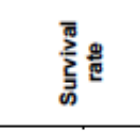 & & 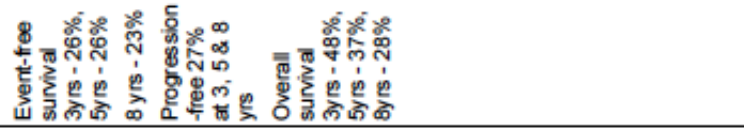 \\
\hline 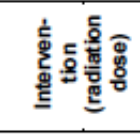 & & 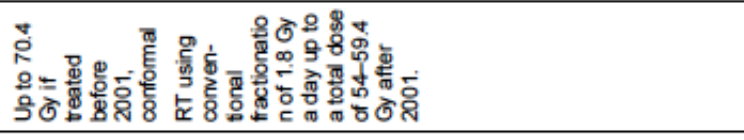 \\
\hline 安䰹 & & 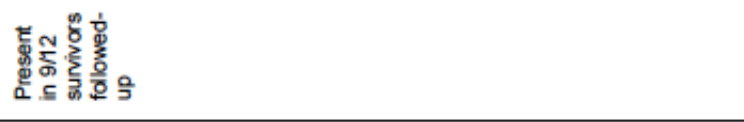 \\
\hline 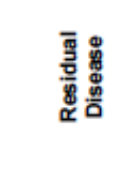 & & 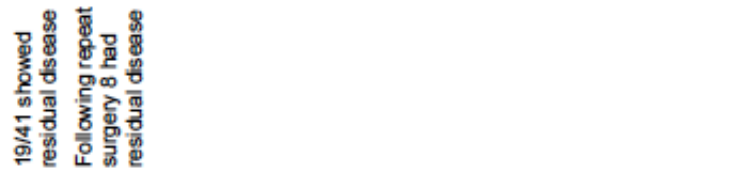 \\
\hline 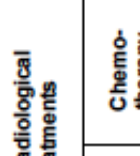 & & 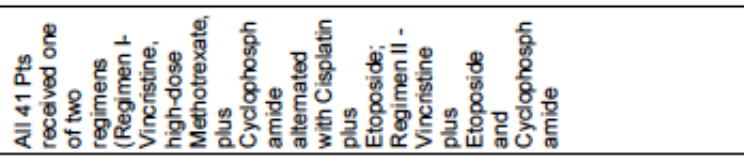 \\
\hline 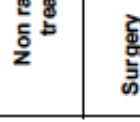 & & 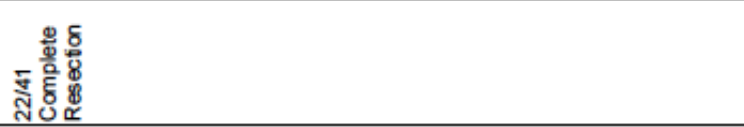 \\
\hline 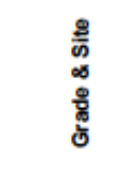 & & 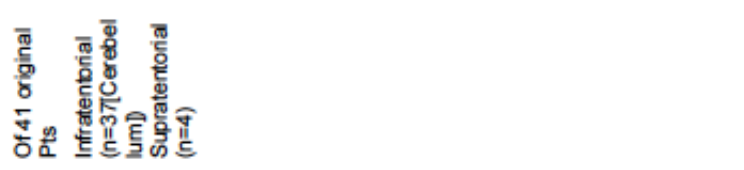 \\
\hline 要 & & 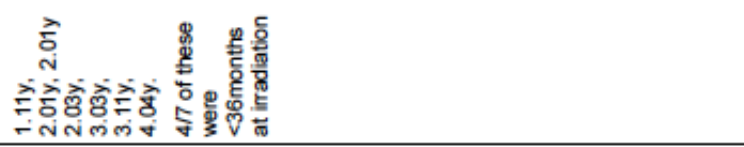 \\
\hline 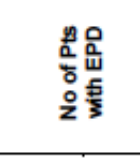 & & 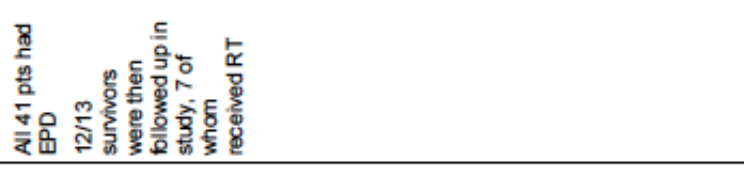 \\
\hline$\frac{\dot{s}}{\underline{\underline{c}}}$ & & 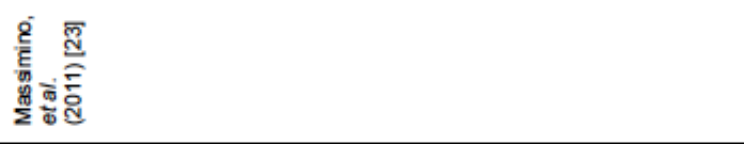 \\
\hline
\end{tabular}




\begin{tabular}{|c|c|c|c|c|}
\hline \multicolumn{2}{|c|}{ 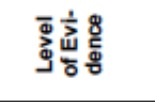 } & & $\stackrel{2}{2}$ & ले \\
\hline & & & 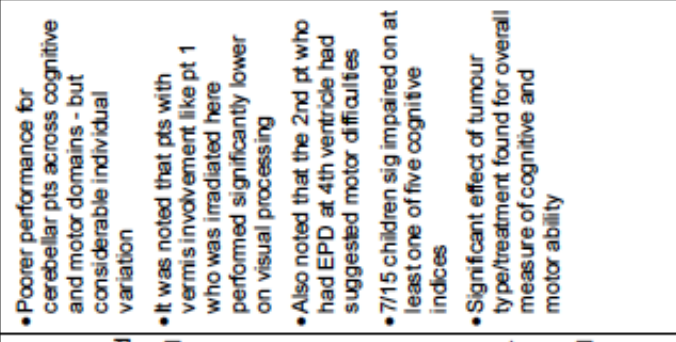 & 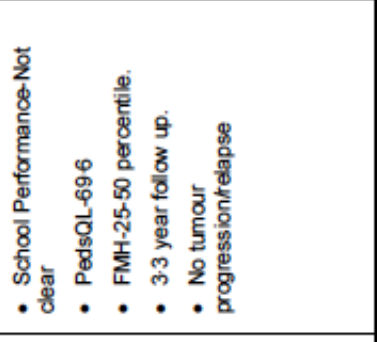 \\
\hline \multicolumn{2}{|c|}{ 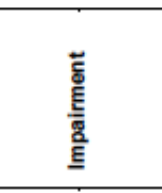 } & 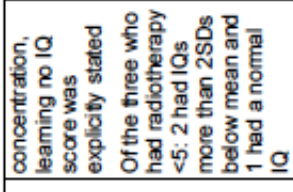 & 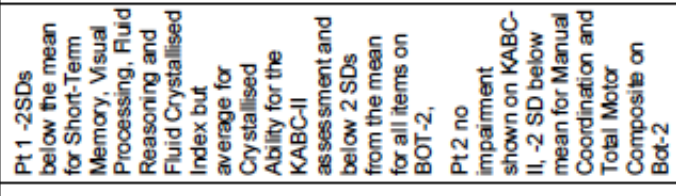 & 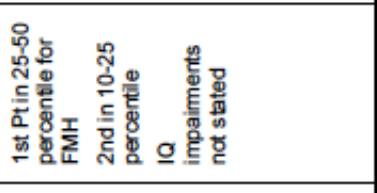 \\
\hline \multicolumn{2}{|c|}{ 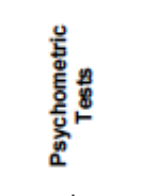 } & & 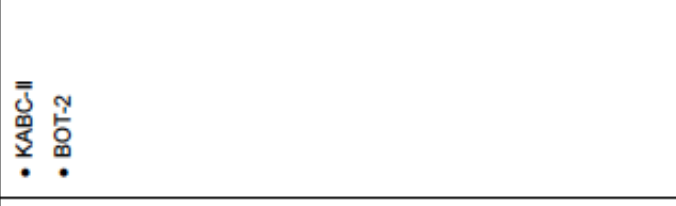 & 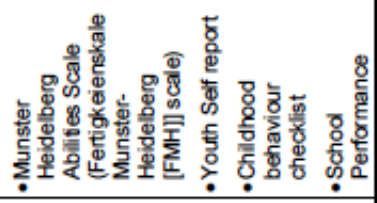 \\
\hline \multicolumn{2}{|c|}{ 要步 } & & 兽 & 额 \\
\hline \multicolumn{2}{|c|}{ 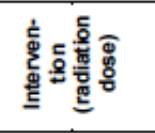 } & & 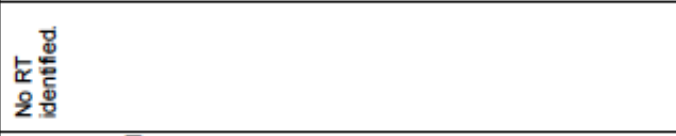 & 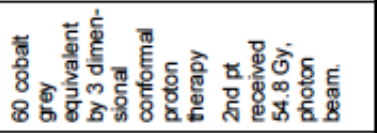 \\
\hline \multicolumn{2}{|c|}{ 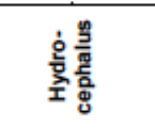 } & & 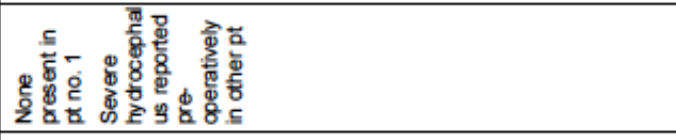 & 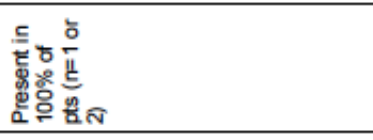 \\
\hline \multicolumn{2}{|c|}{ 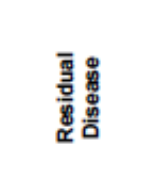 } & & 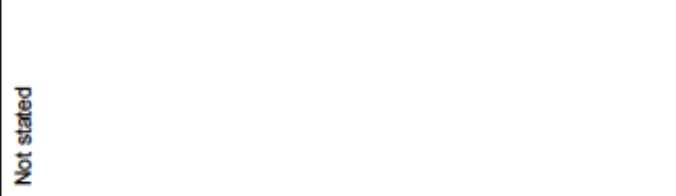 & 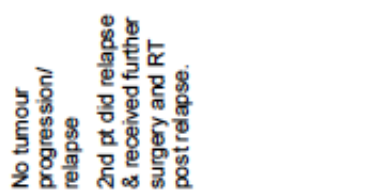 \\
\hline \multirow{2}{*}{ 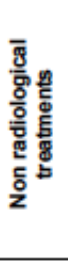 } & 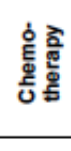 & & 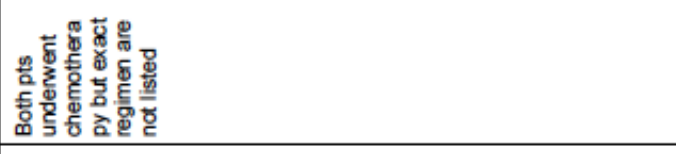 & 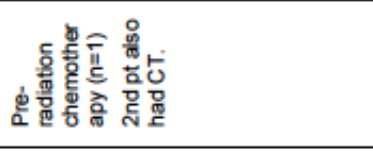 \\
\hline & $\begin{array}{l}\text { 总 } \\
\text { 离 } \\
\end{array}$ & & 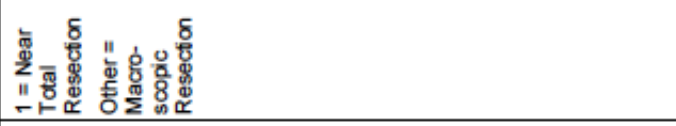 & 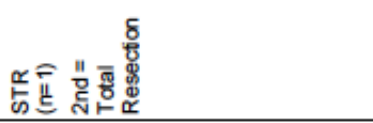 \\
\hline \multicolumn{2}{|c|}{ 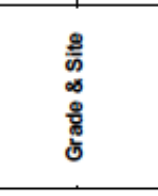 } & & 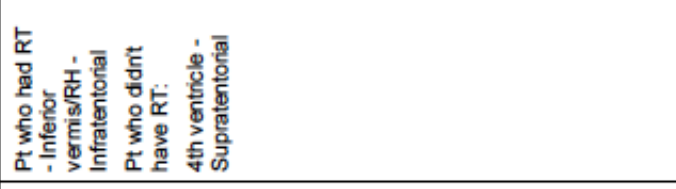 & 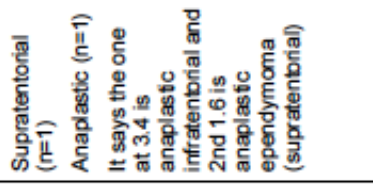 \\
\hline \multicolumn{2}{|c|}{ 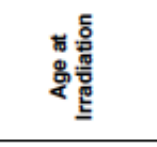 } & & 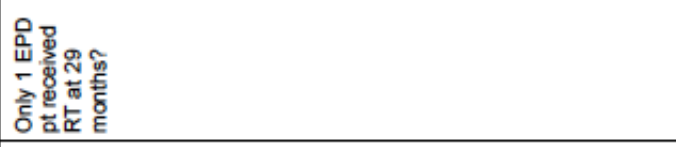 & 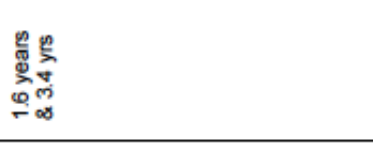 \\
\hline \multicolumn{2}{|c|}{ 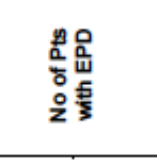 } & & $\stackrel{\frac{1}{2}}{2}$ & 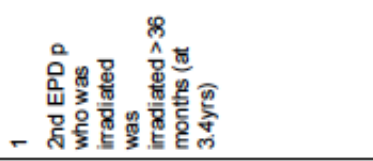 \\
\hline \multicolumn{2}{|c|}{$\frac{\frac{j}{\delta}}{\mathscr{\alpha}}$} & & 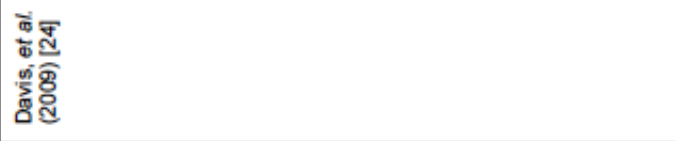 & 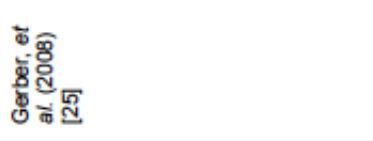 \\
\hline
\end{tabular}




\begin{tabular}{|c|c|c|c|c|c|}
\hline \multicolumn{2}{|c|}{ 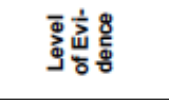 } & & $\stackrel{\pi}{\pi}$ & i & i \\
\hline \multicolumn{2}{|c|}{ 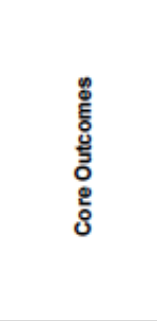 } & & 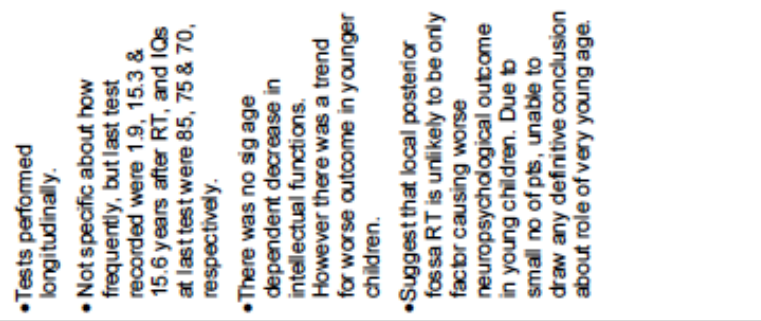 & 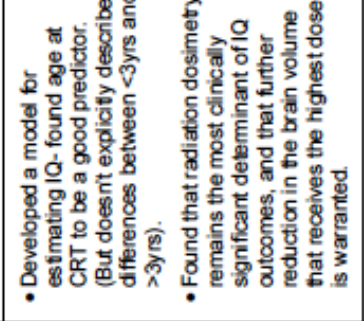 & 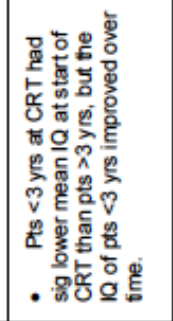 \\
\hline \multicolumn{2}{|c|}{ 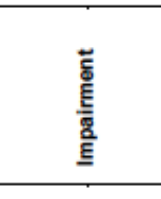 } & & 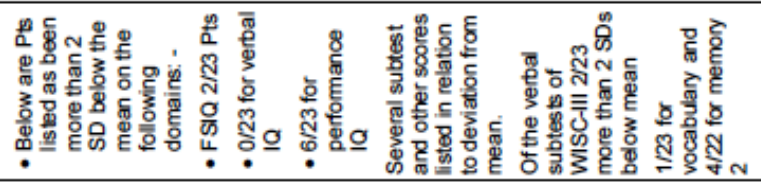 & $\frac{s}{2}$ & 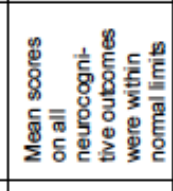 \\
\hline \multicolumn{2}{|c|}{ 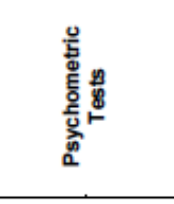 } & $\mid$ & 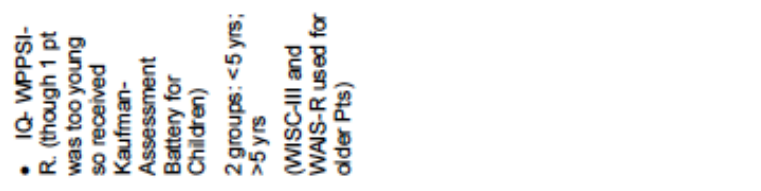 & 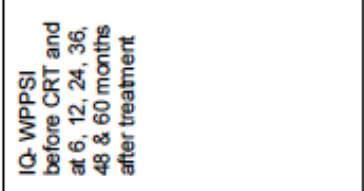 & 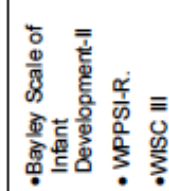 \\
\hline \multicolumn{2}{|c|}{ 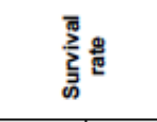 } & & 施 & 草兽 & 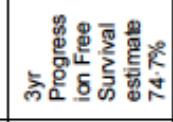 \\
\hline \multicolumn{2}{|c|}{ 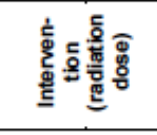 } & & 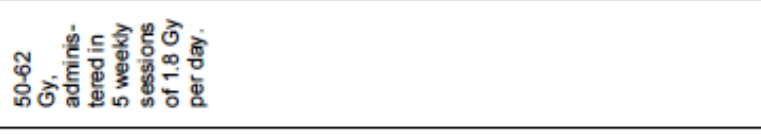 & 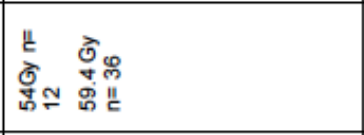 & 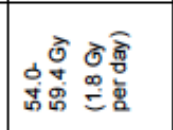 \\
\hline \multicolumn{2}{|c|}{ 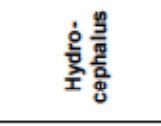 } & & 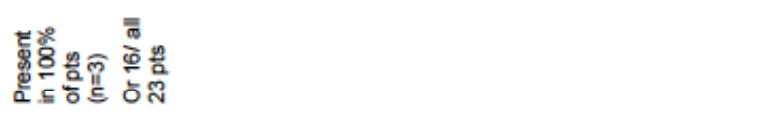 & 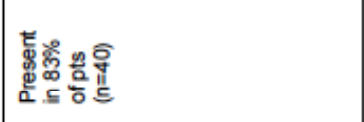 & 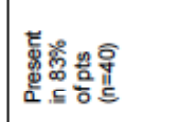 \\
\hline \multicolumn{2}{|c|}{ 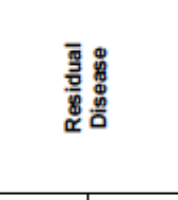 } & & 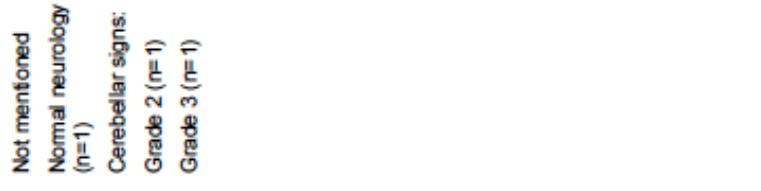 & 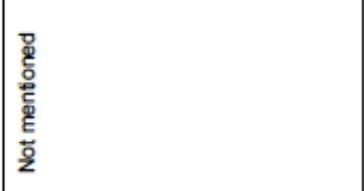 & 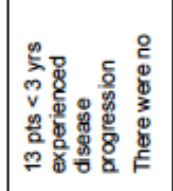 \\
\hline \multirow{2}{*}{ 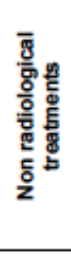 } & 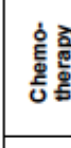 & & 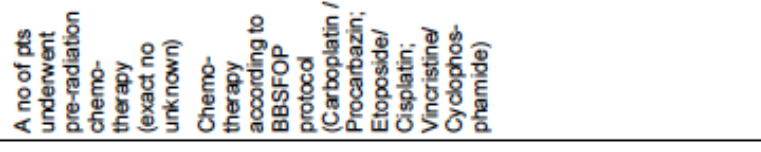 & 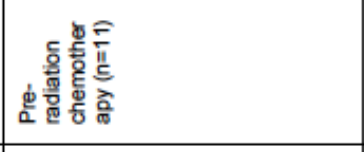 & 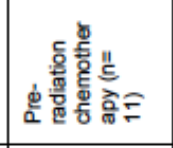 \\
\hline & 总 & & 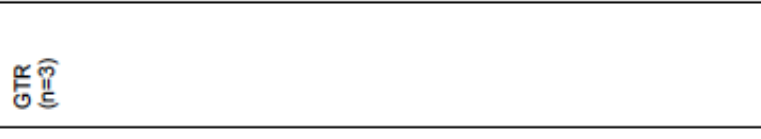 & 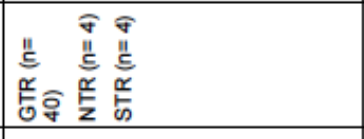 & 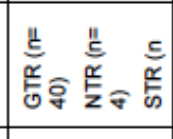 \\
\hline \multicolumn{2}{|c|}{ 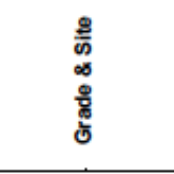 } & & 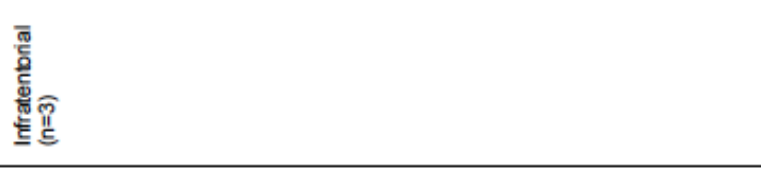 & 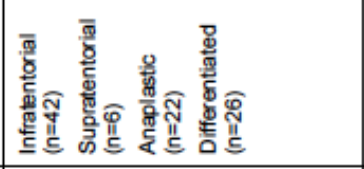 & 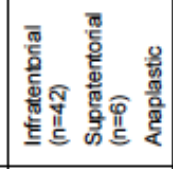 \\
\hline \multicolumn{2}{|c|}{ 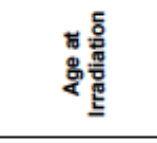 } & & 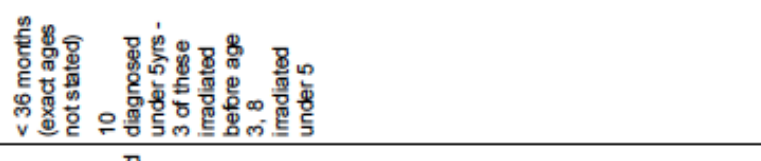 & 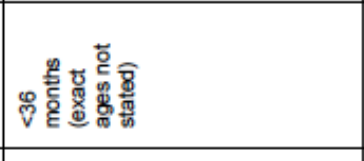 & 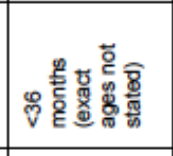 \\
\hline \multicolumn{2}{|c|}{ 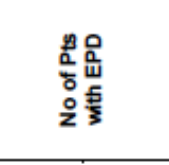 } & & 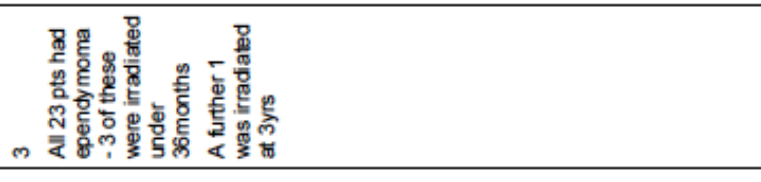 & 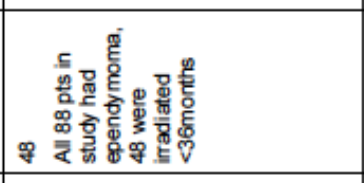 & 娄 \\
\hline \multicolumn{2}{|c|}{$\frac{d}{\frac{\delta}{\alpha}}$} & & 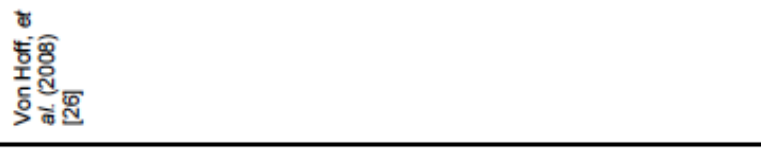 & 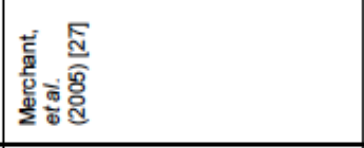 & 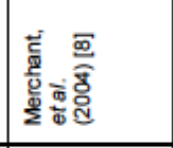 \\
\hline
\end{tabular}




\begin{tabular}{|c|c|c|c|}
\hline \multicolumn{2}{|c|}{ 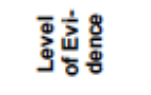 } & & $\stackrel{\sim}{\sim}$ \\
\hline & & 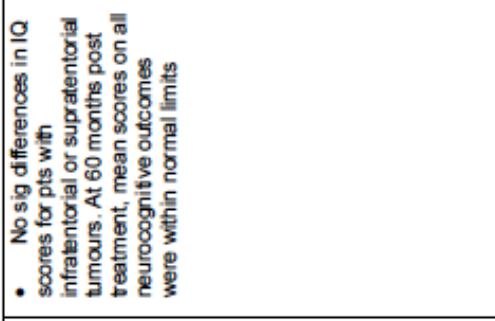 & 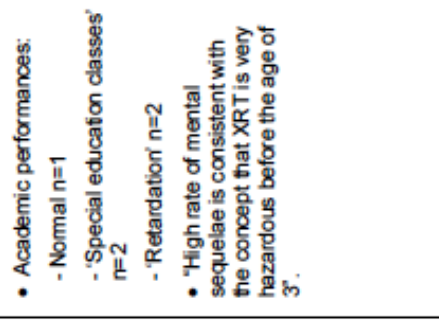 \\
\hline & & 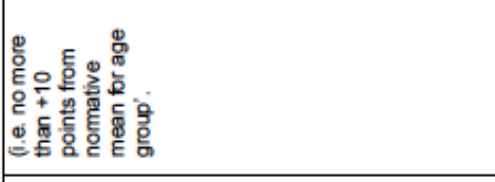 & 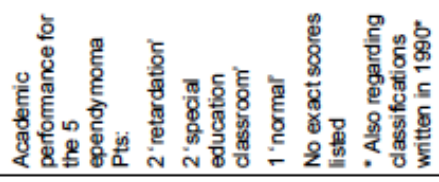 \\
\hline \multicolumn{2}{|c|}{ 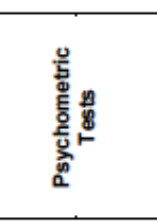 } & 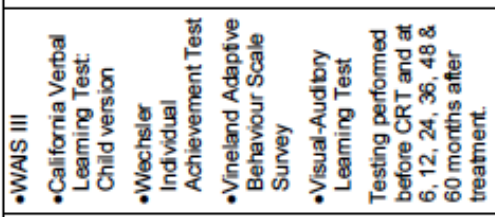 & 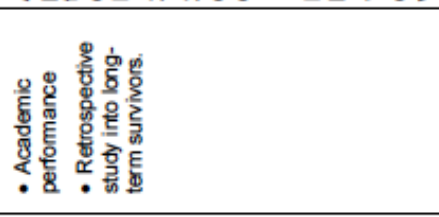 \\
\hline \multicolumn{2}{|c|}{ 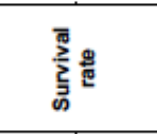 } & 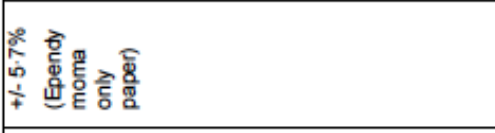 & 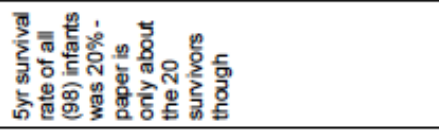 \\
\hline \multicolumn{2}{|c|}{ 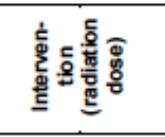 } & & 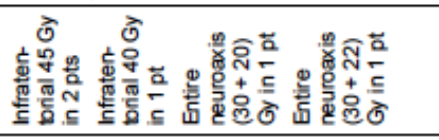 \\
\hline \multicolumn{2}{|c|}{ 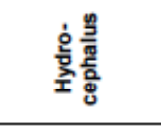 } & & 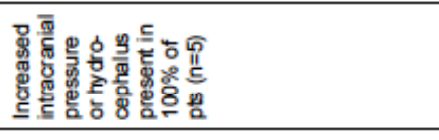 \\
\hline \multicolumn{2}{|c|}{ 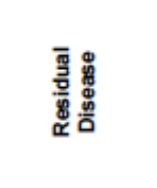 } & 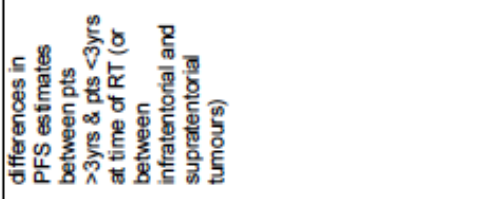 & 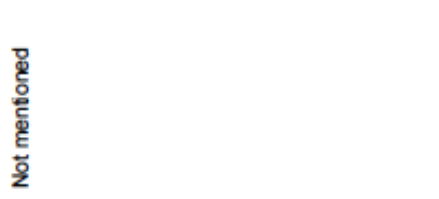 \\
\hline \multirow{2}{*}{ 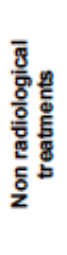 } & 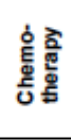 & & 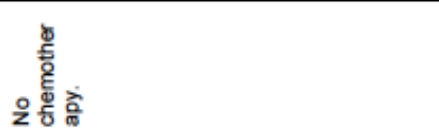 \\
\hline & 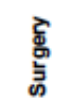 & 栗 & 舀焉总恶 \\
\hline \multicolumn{2}{|c|}{$\begin{array}{l}\frac{8}{5} \\
\text { कू } \\
\text { क्षँ } \\
\text { कू }\end{array}$} & 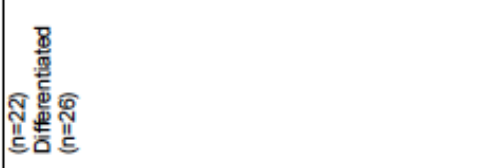 & 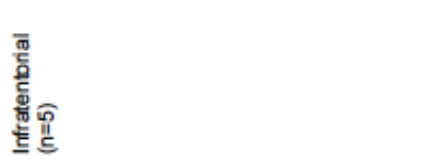 \\
\hline \multicolumn{2}{|c|}{ 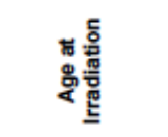 } & & 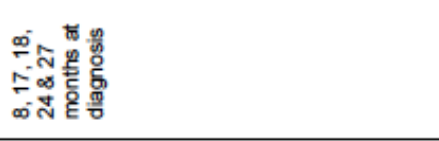 \\
\hline \multicolumn{2}{|c|}{ 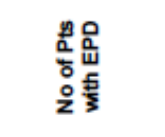 } & & n \\
\hline \multicolumn{2}{|c|}{$\frac{\dot{p}}{\dot{\phi}}$} & & 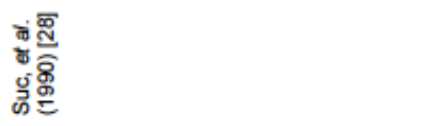 \\
\hline
\end{tabular}

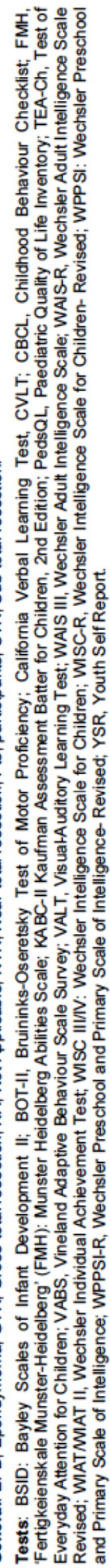




\begin{tabular}{|c|c|c|c|}
\hline 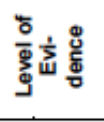 & 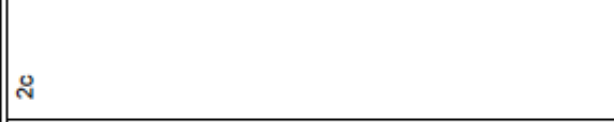 & $\approx$ & i \\
\hline 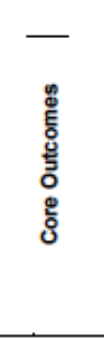 & 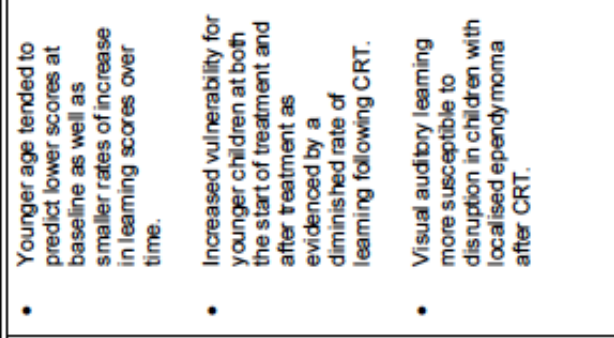 & 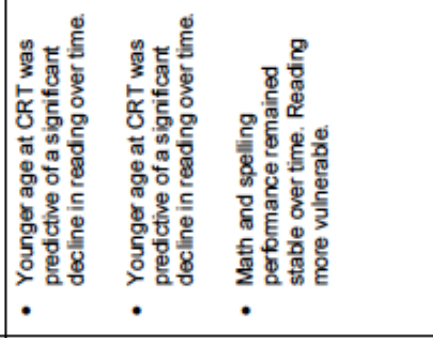 & 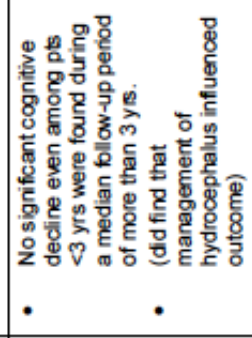 \\
\hline 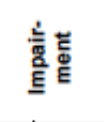 & 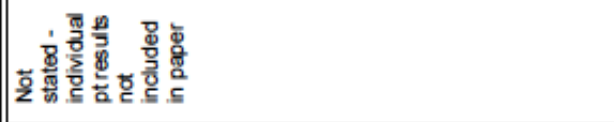 & 竞要 & 童要 \\
\hline 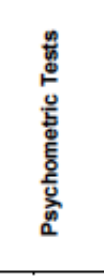 & 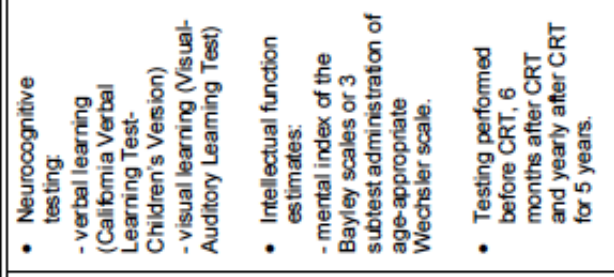 & 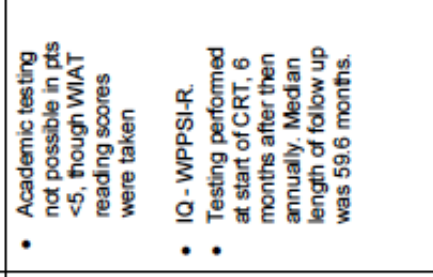 & 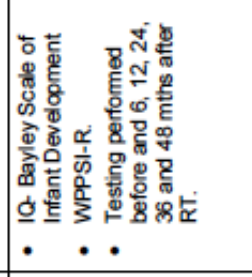 \\
\hline 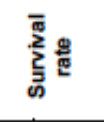 & 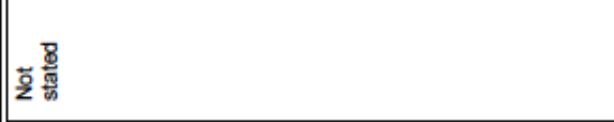 & 搃 & 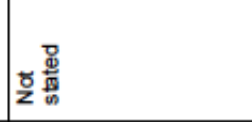 \\
\hline 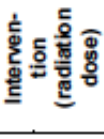 & 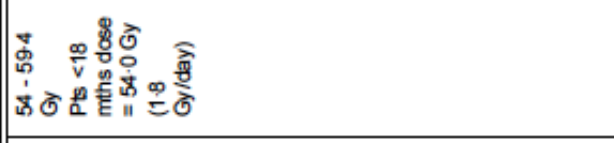 & 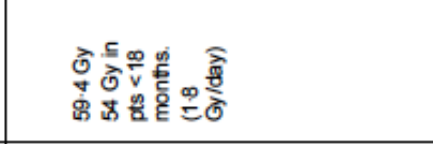 & 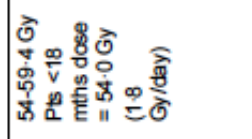 \\
\hline 客墨 & 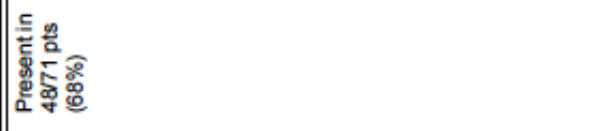 & 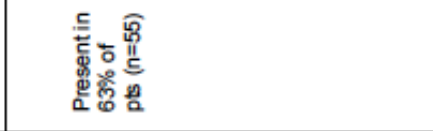 & 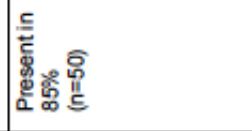 \\
\hline 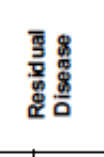 & 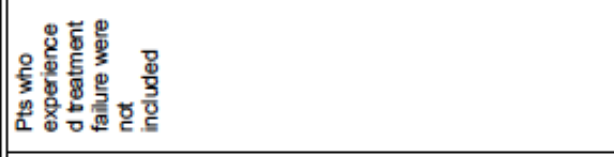 & 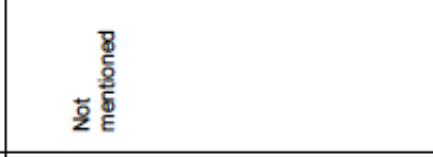 & 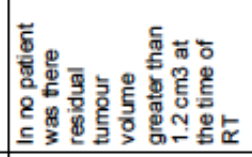 \\
\hline 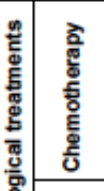 & 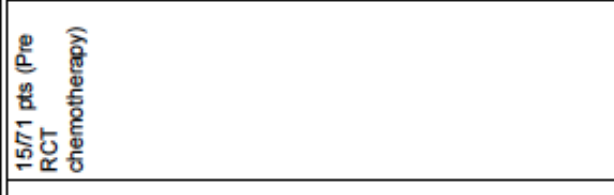 & 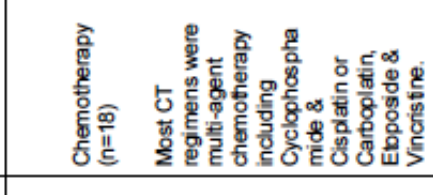 & 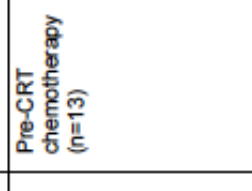 \\
\hline 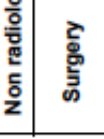 & 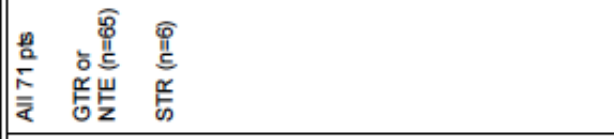 & 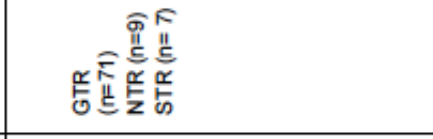 & 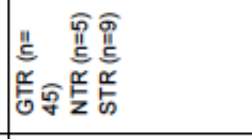 \\
\hline 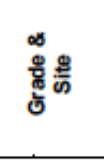 & 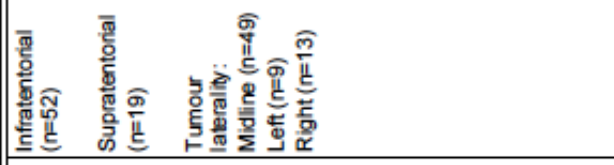 & 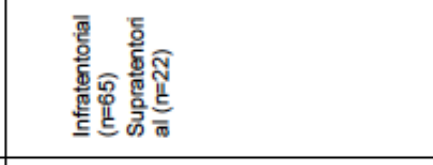 & 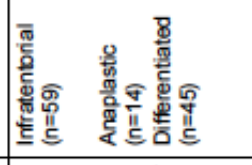 \\
\hline 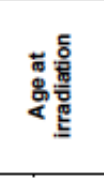 & 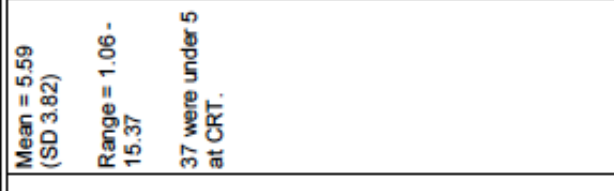 & 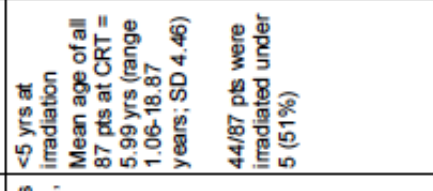 & 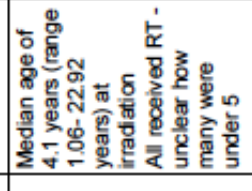 \\
\hline 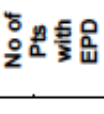 & $\frac{5}{\frac{5}{4}}$ & 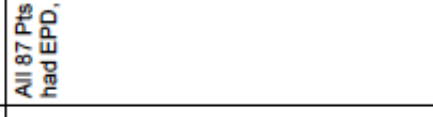 & 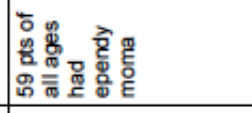 \\
\hline$\frac{\dot{\phi}}{\ddot{\alpha}}$ & 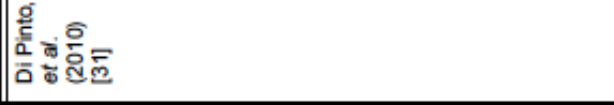 & 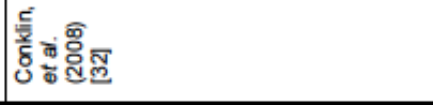 & 喜 \\
\hline
\end{tabular}




\begin{tabular}{|c|c|c|}
\hline 竞离 & $\| \stackrel{\Delta}{*}$ & ले \\
\hline 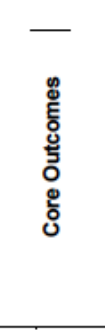 & 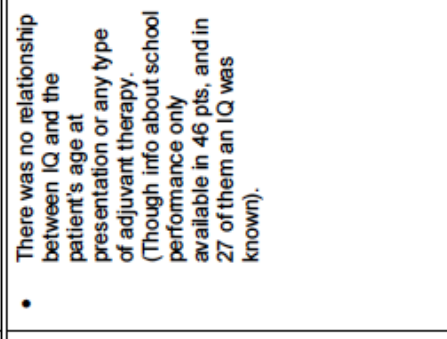 & 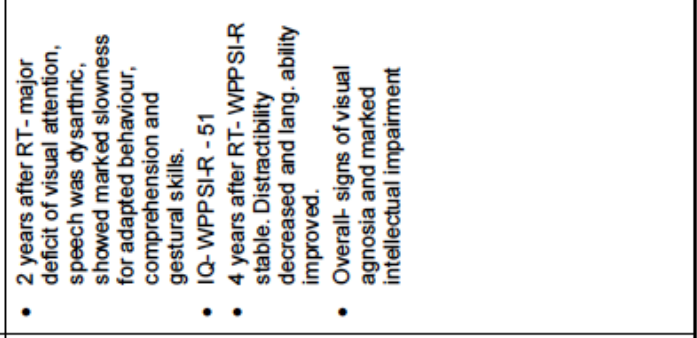 \\
\hline 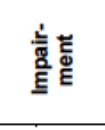 & 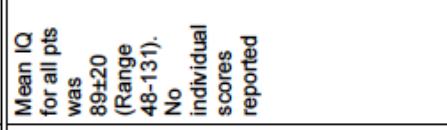 & 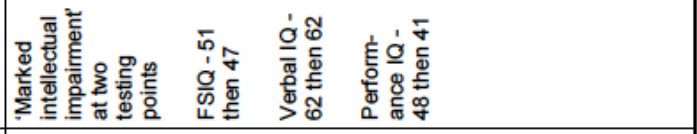 \\
\hline 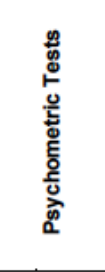 & 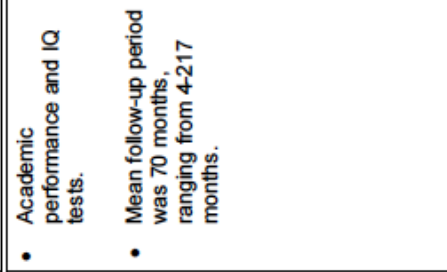 & 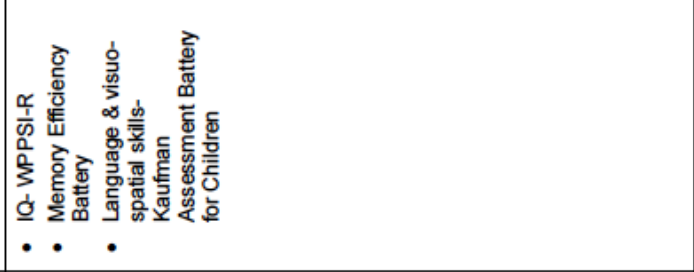 \\
\hline 宽 & 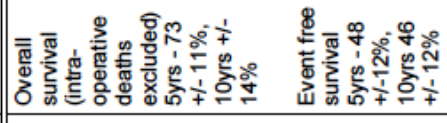 & 응 \\
\hline 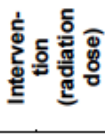 & & 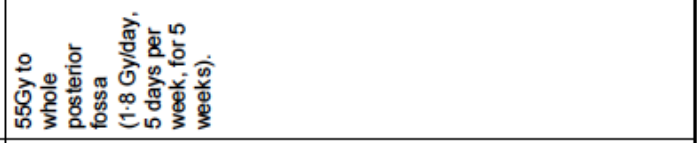 \\
\hline 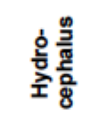 & 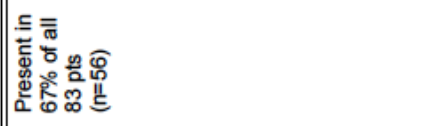 & 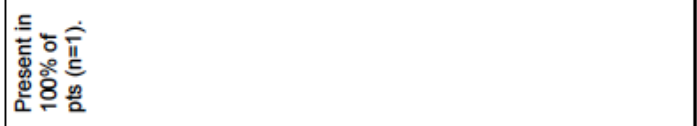 \\
\hline 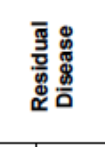 & 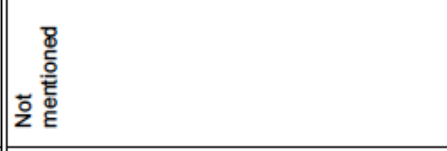 & 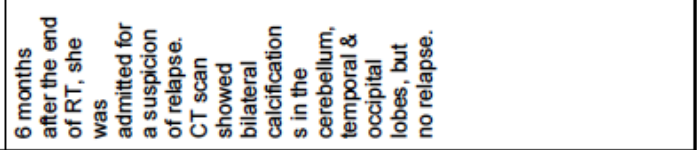 \\
\hline 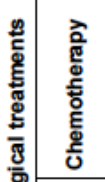 & 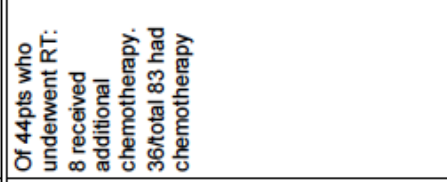 & 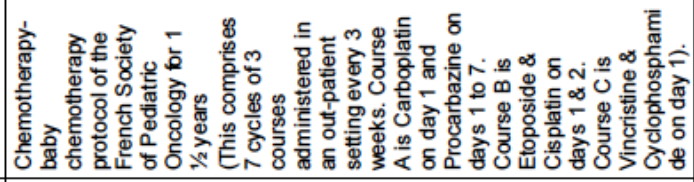 \\
\hline 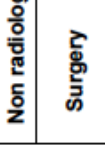 & 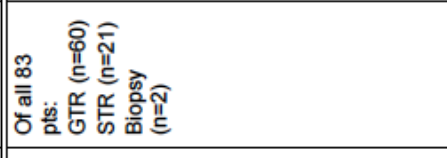 & 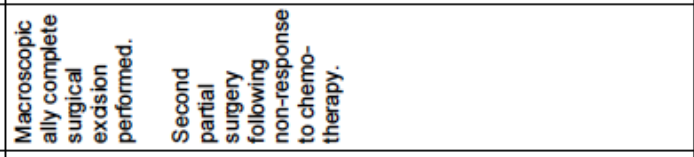 \\
\hline 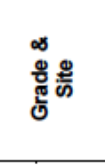 & 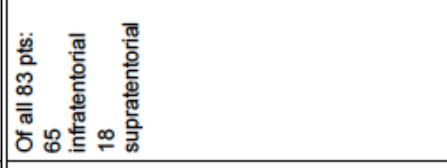 & 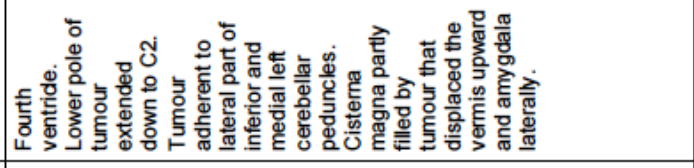 \\
\hline 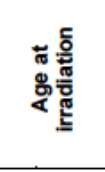 & 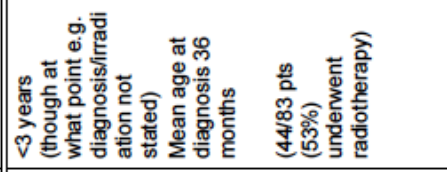 & 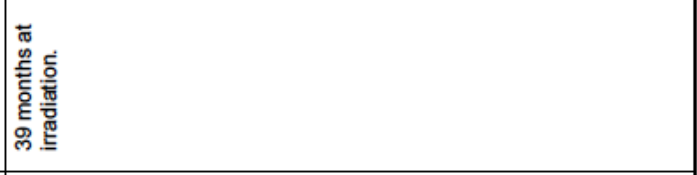 \\
\hline 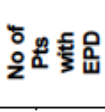 & 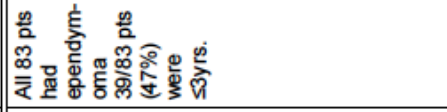 & 흠 \\
\hline 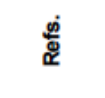 & 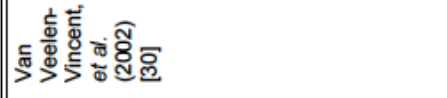 & 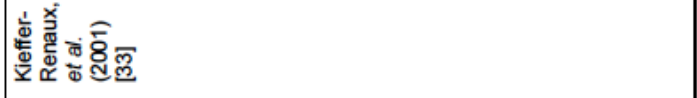 \\
\hline
\end{tabular}

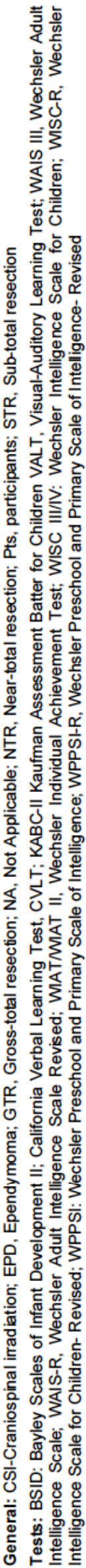


and cyclophosphamide (VEC) +/- second look surgery followed by the described radiotherapy (RT) protocol were given. After 2001, patients with complete resection and Grade II revised histology had CRT with conventional fractionation of $1.8 \mathrm{~Gy} / \mathrm{d}$. Patients with complete excision and Grade III revised histology received four VEC courses after RT. With residual tumour of any grade VEC was given before RT to facilitate second look surgery. In [22, 24] no detailed $\mathrm{RT}$ protocol is described but presence and complexity of hydrocephalus with required treatment is given [22]. For [23] adjuvant treatment was planned to start within four weeks of surgery and followed two different treatment protocols. In regimen I (1994-2003) four blocks of vincristine $\left(1.5 \mathrm{mg} / \mathrm{m}^{2}\right)$ plus high-dose methotrexate $5 \mathrm{~g} / \mathrm{m}^{2}$ with cyclophosphamide $1.5 \mathrm{~g} / \mathrm{m}^{2}$ alternating with cisplatin $90 \mathrm{mg} / \mathrm{m}^{2}$ plus VP16 450 $\mathrm{mg} / \mathrm{m}^{2}$ for year one. Regimen II included VEC: VCR $1.5 \mathrm{mg} / \mathrm{m}^{2}$ plus VP16 $300 \mathrm{mg} / \mathrm{m}^{2}$ and CTX $3 \mathrm{~g} / \mathrm{m}^{2}$ for six months. CT was discontinued following disease progression. RT was planned only for patients with residual tumour after CT or progression of tumour while receiving CT. RT doses and schedules varied according to the used protocol: hyperfractionated RT (1.1 Gy twice a day) administered to the tumour bed with a 1-2 $\mathrm{cm}$ margin (margin reduction was adopted during that time according to physicians' experience and literature), up to $70.4 \mathrm{~Gy}$ for children treated before 2001, or conformal RT using conventional fractionation of 1.8 Gy a day up to a total of 54-59 Gy after 2001. Post operative and pre-irradiation MRI defined the residual disease and possibly collapsed post-surgical tumour bed. The planning target volume was $0.5 \mathrm{~cm}$ larger than the clinical target volume in all directions. No reduction of fields or radiation boost was planned in case of residual tumour. No detailed information regarding $\mathrm{RT}$ is provided in [25]. For [26] total dose ranged from 50-62 Gy, administered in five weekly sessions of 1.8 Gy per day. For patients early in the series, radiographic simulation images with handdrawn tailored shielding based upon physician knowledge of anatomical structures and tumour characteristics were used. For those treated later, 3D high definition CT-based representation of dosedistribution superimposed with posterior fossa structures and tumour contour were available. The GTV for the primary site boost included the postoperative tumour bed. The CTV included in the GTV with an anatomically confined margin of $2 \mathrm{cms}$ in the adjacent brain whereas the PTV expanded the CTV with a geometric margin of $1 \mathrm{~cm}$. Multiple beam arrangements were used. Their initial approach induced full dose to the entire posterior fossa including occipital and posterior temporal areas. Only the pituitary area located at the anterior margin was kept to an 'acceptable' level. The later approach permitted reduced maximal dose to most structures outside the posterior fossa. Papers $[8,27]$ present the same patients. The GTV contained the tumor bed, residual tumor, or both. The CTV contained the GTV with an added margin of $1 \mathrm{~cm}$, which was included so that subclinical microscopic disease beyond the GTV could be treated. The CTV was anatomically confined; that is, it was limited by normal tissue structures through which tumor extension was unlikely. The planning target volume included the CTV surrounded by an additional margin of 3 to $5 \mathrm{~mm}$, expanded in three dimensions to account for uncertainty in patient positioning and image registration. Conventional fractionation (1.8 Gy per day) was used to treat all patients, and the prescribed dose was $59.4 \mathrm{~Gy}$. Exceptions included children younger than 18 months and three children older than 18 months who received 54.0 Gy after gross-total resection. For [28] minimal data regarding $\mathrm{RT}$ is provided.

\section{Mortality and Neurocognitive Morbidity}

Typically, survival rates were not stated. When they were included $(33.3 \%$; $3 / 9)$ the calculation had been completed for all patients (of any age at irradiation or any tumour type in mixed studies) and ranged from $20 \%$ at five years [28] and $74.7 \%$ at three years [8]. A total of 13 different psychometric tests were used (excluding editions of the same test e.g. WISC III and WISC IV were classed as one test, three of which were proxy measures - CBCL, PedsQL and VABS). Five studies used Wechsler ability measures (WPPSI, WPPSI-R, WISC-III, WISC-IV, WAIS-R and WAIS-III to obtain IQ $[7,8,22,26,27]$. Three studies [23,25,28] reported IQ scores and/or scholastic performance with no indication as to how this was obtained. One study stated that patients who were not irradiated did not demonstrate better outcomes than those who were [23]. Another indicated that radiation dosimetry was the most clinically significant determinant of IQ outcome [27] with a further [28] agreeing that radiation before 36 months was 'very hazardous' for mental sequelae. One study suggested that radiotherapy was unlikely to be the only factor contributing to poor neurocognitive outcome in young children [26]. A further paper [7] suggested that tumour location and pre-/perioperative damage seemed to affect cognitive outcome more than age at RT. 


\section{DISCUSSION}

This systematic literature review has identified only limited data from published studies regarding morbidity and mortality of post surgical irradiation. There is significant scope to develop a better evidence base and improve neurocognitive assay.

Sixty-six children under 36 months received radiotherapy with $80 \%(53 / 66)$ of these children receiving infratentorial radiotherapy and 14\% (9/66) supratentorial radiotherapy. For the remaining children, anatomical site was not specified. One child was irradiated (infratentorial) at $<12$ months. Of 14 papers reaching minimum quality standards, nine papers indicated radiotherapy for childhood ependymoma leads to lower IQ scores or poorer overall cognitive outcome [22, 27, 28, 31, 33, 35, 37, 42, 44] compared to norms. One of these studies [28] suggested that young age at CRT is a further risk factor with Di Pinto et al. [31] stating that young age at irradiation leads to smaller rates of increase in learning over time and Kieffer-Renaux et al. [37] noting that IQ continues to decline more than four years post diagnosis. Conklin et al. [32] identify that young age at CRT affects reading ability with Pulsifer et al. [44] finding significant decline in processing speed and visual-spatial organisation in childhood ependymoma survivors. In contrast, six of the retrieved papers stated CRT does not predict poorer cognitive outcomes [7,23,29,30,36,41]. Further to this, Merchant and colleagues [8] state that being less than 36 months old at time of radiotherapy may lead to lower IQ but that this is a product of the tumour itself and following CRT, cognition may improve over time. It is important to note that improvements may well occur but as a consequence of the normal neurodevelopmental process. What remains unclear is whether the rate of new learning and skill acquisition post CRT is commensurate with typical cognitive trajectories. Poggi et al. [39] found that young age (0$6 y$ rs) at radiotherapy leads to lower cognitive impairment. Young age and CRT may not be the only factors leading to a reported decline in cognitive function. For example, cognitive deficits or low IQ may be predicted by radiation dosimetry [27], tumour location [7,42]; pre- or perioperative brain damage [7] or presence of lacunae [38]. The presence and management of hydrocephalus are also implicated as factors effecting cognitive outcome [29]; however, Davis et al. [22] did not replicate this finding with no consistent effect of hydrocephalus on outcome demonstrated. Where IQs are reported, large inter- individual differences [22] were present with no definitive explanation provided accounting for this variability.

Twenty-five papers were found to include childhood ependymoma patients who had received radiotherapy as treatment. In comparison to the wealth of studies available for mortality rates, there is a paucity of work describing cognitive morbidity for irradiated survivors of childhood ependymoma. Of the few studies that investigated this and are consequently included in this review, a majority were rated at $2 b$ for quality of evidence [20]. In all but one of the twenty-five studies reviewed, the number of ependymoma patients could be identified clearly. However, determining patient age at diagnosis, treatment or follow-up was not straightforward. Scrutiny of retrieved papers led to three categories of data emerging. Nine papers (Table 2) stated explicitly that patients were irradiated for ependymoma at $<36$ months. Five references (Table $\mathbf{3}$ ) included patients who received radiotherapy for an ependymoma at $<60$ months. Therefore, some of these patients may have been $<36$ months but this information could not be ascertained. Finally, eleven papers (Supplementary Table) presented children who were treated with radiotherapy for ependymoma but age was not specified. Data were of variable quality. Where ependymoma patients were clearly identifiable their numbers ranged from 1-88. Those that included ependymoma patients only led to more accessible data. In papers where more than one brain tumour type was discussed, data regarding irradiation outcomes for ependymoma were more difficult to access.

Methodological limitations are present in the retrieved papers. There is inconsistency for data reporting ensuring comparisons and more standard forms of statistical scrutiny cannot presently be performed. The use of psychometry was an inclusion criterion for papers in this review and, therefore, all papers discussed make reference to some form of neurocognitive assessment and outcome. However, there are inconsistencies across the retrieved papers for the measures used and the way in which obtained results were reported. Across all studies, 16 different measures were used to explore neurocognitive functioning in differing combinations. Some commonality occurs with $69 \cdot 6 \%(18 / 25)$ of papers using a Wechsler test to establish IQ. In three studies IQ is stated but no information is given regarding how this was obtained. Four papers discuss vague descriptions of scholastic outcomes. 
Comparisons cannot be made across all papers, as there is a lack of sufficient data delineation and stratification. Some papers (e.g. [22]) compare the outcomes of irradiated ependymoma survivors according to neurological results such as 'presence/absence' of hydrocephalus. They also include the numbers of patients who received radiotherapy but do not compare results according to treatment received, possibly due to small sample size. Hydrocephalus has been identified as a potential risk factor for cognitive decline following a brain tumour such as ependymoma [29] but its presence or absence was only reported in $68 \%(17 / 25)$ of the studies. Other reasons for poor outcomes are included within studies variably. For example, radiation dosimetry is well reported (22/25 studies) as is tumour location in $19 / 25$. The main issue with this information is that it cannot be specifically identified for ependymoma patients and, therefore, conclusions cannot be drawn. Publishing of individual data via supplementary tables may help to improve analysis to ensure accurate neurocognitive prognosis for this group. The benefits of this approach have been demonstrated with other neurocognitively impaired paediatric groups e.g. $[45,46]$.

Given the likely role of the cerebellum in cognitive development and the demonstrated variability in the neurocognitive outcomes for this group, it is not presently possible to be confident that these children will be unaffected in the long term. Current evidence indicates the cerebellum is involved in the construction and organisation of higher cognitive functions and social behaviours [47] typically associated with the prefrontal cortex. This reflects the integrated network of neural inputs into the cerebellum from all levels of the CNS, including spinal, vestibular and cerebral pathways. Damage to the cerebellar hemispheres has been shown to be associated with intellectual changes, with damage to the vermis associated with behavioural changes [13]. Reciprocal projections between the cerebellum and cerebral cortex provide a plausible neuroanatomical basis for a cerebellar role in cognition [47]. While damage to either cerebellar hemisphere produce ipsilateral motor deficits, projections from the cerebellum to the cerebral cortex are contralateral. Consistent with this structural organisation, evidence indicates lateralized cerebellar lesions produce cognitive deficits similar to those observed following lesions of the contralateral cerebral hemisphere [48]. It is hypothesized that this may be caused by disruption of the metabolic activity to cerebello-cortical pathways $[49,50]$. Therefore, verbal functions and/or literacy defi- cits, in right-handed individuals, have been associated with right cerebellar damage and visuospatial deficits with left cerebellar damage [13]. Because of the increasing role attributed to the cerebellum in higher cognitive functions [15] and acquisition of literacy $[12,14]$, cerebellar dysfunction secondary to the tumour and its treatment(s) is implicated as having a major detrimental effect on intellectual, cognitive, learning and functional outcomes [51].

Although the retrieved papers testify to the importance of assessing neurocognitive outcomes, it is critical to note that no clear neurodevelopmental model is ever presented to account for the findings. This is concerning as its omission limits a complete and longterm understanding of cognitive development and its impairment or indeed resilience for this group of children. The timing of acquired damage, the period of cognitive development and brain maturation all provide the potential for demonstrated adverse 'downstream effects' on yet to be acquired skills, such as literacy and later cognition [52]. The recognition of a primary damage leading to later manifesting secondary impairments ensures the need for long-term prospective surveillance of neurocognitive outcomes. For example, as modest associations exist between developmental tests and later IQ [53], it is inappropriate to draw definitive conclusions regarding patients' likely cognitive abilities and learning outcomes in later life from measures used in early childhood. In addition, the maximum length of follow-up for ependymoma patients was 60 months post treatment [8]. Thus, if the patient was 36 months when receiving radiotherapy their maximum age at follow-up would be eight years. This period of follow up has created the claim [3] that learning in these children remain unaffected. 'Mechanical' literacy skills i.e. reading accuracy and spelling, continue to develop beyond eight years of age [54] with the comprehension of read materials becoming increasingly important. Some papers (e.g. [8]) provide the mean scores for the reading accuracy and spelling components of literacy. Reading comprehension remains unassessed. A child learns to read, then reads to learn. If acquisition of literacy is impaired then all that flows from this will be affected similarly. Impaired literacy acquisition across childhood can adversely affect IQ in the long-term [55]. From Table 2, only 4/9 studies examine literacy in different and incomplete ways. Given the evidence for cerebellar involvement in the acquisition of literacy, more detailed prospective assay of reading is now required. Cognition and learning continue to unfold beyond eight years of 
age and outcomes beyond this remain unknown. In addition, there is evidence that children who are initially assessed as without difficulties may develop significant later, more global, impairments to cognitive functioning due to the phenomenon of 'growing into deficit' [56]. With improved follow-up and consistent neurocognitive assay, treating and research communities may be better able to substantiate the claim for an absence of adverse neurocognitive sequelae for irradiation at $<36$ months. While a complete absence of late neurocognitive effects may not be a realistic aim, the aim to address methodological variation and inconsistent capture of neurocognitive outcome is.

Sample size varies and data collection is retrospective or prospective. The technique of RT used; timing of $\mathrm{RT}$; role of multiple surgery and presence of cerebellar mutism are described variably. In [8] patients were treated with post-surgical RT for initial management. For [23] most received RT as part of a salvage strategy including repeat surgery. Multiple resection and anticipated and non-anticipated post-neurosurgical complications may restrict clarity of conclusion further. Given the variability of data presentation and differing opinions regarding the role of RT in neurocognitive sequelae it is recommended that data capture should be standardised. To better establish the longer-term risk for this group, data collection for the following are suggested: presence of cerebellar mutism, tracheostomy rates; vascular events; number of days in PICU; number of surgeries performed; presence of residual disease; premorbid difficulties; ability and literacy outcomes, using Wechsler tests.

Claims $[8,19]$ for the absence of long-term neurocognitive impairment in childhood ependymoma (3 years of age) require further evaluation as retrieved evidence questions this view. From retrieved evidence, considerable variability in neurocognitive outcome is demonstrated for children who received radiotherapy for ependymoma at this age. The retrieved papers raise the question of the type of data needed by the treating and research community to fully understand the long-term neurocognitive consequences of ependymoma and their treatments. Without this, the actual morbidity and the full costs of long term neurodisability, unemployment and underemployment will never be known. This paper only reviews the reported neurocognitive sequelae of photon radiotherapy for young ependymoma patients. As proton radiotherapy is increasingly being used it is important to address consistency of methodology and data reporting. Although at present it may not be possible to achieve consensus for international clinical practice, it is crucial to establish a common agreement for study design; neurocognitive development, learning and its measurement; consistency and delineation of data capture and reporting, and duration of follow-up, to allow systematic comparisons across studies to be made. The International Society for Paediatric Oncology (SIOP) is currently working towards this.

\section{CONFLICT OF INTEREST}

No conflict of interest is declared.

\section{FUNDING}

Candlelighters, Children's Brain Tumour Research Centre; Joseph Foote Foundation.

\section{ACKNOWLEDGEMENTS}

The authors wish to thank the Candlelighters, Children's Brain Tumour Research Centre, Joseph Foote Foundation, Prof. Haggar, Miss Reed-Berendt and Drs. Livingston and Picton.

\section{REFERENCES}

[1] Kulkarni AV, Bouffet E, Drake J. Ependymal tumors. In: Walker DA, Perilongo G, Punt JAG. \& Taylor RE (eds.) Brain and Spinal Tumors of Childhood. London: Arnold 2004; p. 331-344.

http://dx.doi.org/10.1201/b13511-25

[2] Grundy RG. Primary radiotherapy for childhood ependymoma? Lancet Oncol 2007; 8(9): 760-1. http://dx.doi.org/10.1016/S1470-2045(07)70255-3

[3] Heidemann RL PR, Albright LA, Freeman CR, Rorke LB. Tumors of The Central Nervous System. 1st edition ed. Philadelphia: Lippincott-Raven 1997; p. 633-697.

[4] Duffner PK, Krischer JP, Sanford RA, et al. Prognostic factors in infants and very young children with intracranial ependymomas. Pediatr Neurosurg 1998; 28(4): 215-22. http://dx.doi.org/10.1159/000028654

[5] Sala F, Talacchi A, Mazza C, Prisco R, Ghimenton C, Bricolo A. Prognostic factors in childhood intracranial ependymomas: the role of age and tumor location. Pediatr Neurosurg 1998; 28(3): 135-42.

http://dx.doi.org/10.1159/000028637

[6] Kilday JP, Rahman R, Dyer S, Ridley L, Lowe J, Coyle B, et al. Pediatric ependymoma: biological perspectives. Mol Cancer Res 2009; 7(6): 765-86. http://dx.doi.org/10.1158/1541-7786.MCR-08-0584

[7] Poggi G. Adduci A, Gandola L, Galbiati S, Degrate A, Sironi $\mathrm{E}$, et al. Cognitive and psychological outcomes in younger vs. older children with subtentorial/supratentorial ependymoma after radiation therapy. J Behav Brain Sci 2011; 1: 87-93. http://dx.doi.org/10.4236/jbbs.2011.13012

[8] Merchant TE, Mulhern RK, Krasin MJ, Kun LE, Williams T, L $\mathrm{C}$, et al. Preliminary results from a phase II trial of conformal radiation therapy and evaluation of radiation-related CNS effects for pediatric patients with localized ependymoma. $J$ Clin Oncol 2004; 22(15): 3156-3162.

http://dx.doi.org/10.1200/JCO.2004.11.142 
[9] Grundy RG, Wilne SA, Weston CL, Robinson K, Lashford LS, Ironside $\mathrm{J}$, et al. Primary post-operative chemotherapy without radiotherapy for intracranial ependymoma in children: the UKCCSG/SIOP prospective study. Lancet Oncol 2007; 8(8): 696-705.

http://dx.doi.org/10.1016/S1470-2045(07)70208-5

[10] Patel SK, Mullins WA, O'Neil SH, Wilson K. Neuropsychological differences between survivors of supratentorial and infratentorial brain tumours. J Intellect Disab Res 2011; 55(1): 30-40. http://dx.doi.org/10.1111/j.1365-2788.2010.01344.x

[11] Dolan RJ. Editorial: A cognitive affective role of the cerebellum. Brain 1998; 121: 545-546. http://dx.doi.org/10.1093/brain/121.4.545

[12] Stoodley CJ, Stein JF. The cerebellum and dyslexia. Cortex 2011; 47: 101-227.

http://dx.doi.org/10.1016/j.cortex.2009.10.005

[13] Scott RB, Stoodley CJ, Anslow P, Paul C, Stein JF, Sugden $\mathrm{EM}$, et al. Lateralized cognitive deficits in children following cerebellar lesions. Develop Med Child Neurol 2001; 43: 685691.

http://dx.doi.org/10.1017/S0012162201001232

[14] Nicolson RI, Fawcett AJ, Dean P. Developmental dyslexia: the cerebellar deficit hypothesis. Trends Neurosci 2001; 24 (9): 508-511. http://dx.doi.org/10.1016/S0166-2236(00)01896-8

[15] Diamond A. Close interrelation of motor development and cognitive development and of the cerebellum and prefrontal cortex. Child Develop 2000; 71(1): 44-56. http://dx.doi.org/10.1111/1467-8624.00117

[16] Broniscer A, Ke W, Fuller CE, Wu J, Gajjar A, Kun LE. Second neoplasms in pediatric patients with primary central nervous system tumors: the St. Jude Children's Research Hospital experience. Cancer 2004; 100(10): 2246-52. http://dx.doi.org/10.1002/cncr.20253

[17] Lannering B, Marky I, Lundberg A, Olsson E. Long-term sequelae after pediatric brain tumors: their effect on disability and quality of life. Med Pediatr Oncol 1990; 18(4): 304-10. http://dx.doi.org/10.1002/mpo.2950180410

[18] Spoudeas $\mathrm{H}$, Kirkham F. Toxicity and late effects. In: Walker DA, Perilongo G, Punt JAG, Taylor RE, eds. Brain and Spine Tumours of Childhood. London: Arnold 2004; 433-62. http://dx.doi.org/10.1201/b13511-34

[19] Bouffet E, Tabori U, Bartels U. Paediatric ependymomas: should we avoid radiotherapy? Lancet Oncol 2007; 8; 665666. http://dx.doi.org/10.1016/S1470-2045(07)70213-9

[20] Oxford Centre for Evidence-based Medicine. Levels of Evidence March 2009 [Cited 2011 July 22]. Available from: http://www.cebm.net/index.aspx?o=1025

[21] Moher D, Liberati A, Tetzlaff J, Altman DG, The PRISMA Group (2009). Preferred Reporting Items for Systematic Reviews and Meta-Analyses: The PRISMA Statement. PLoS Med 6(6): e1000097. doi:10.1371/journal.pmed1000097

[22] Davis EE, Pitchford NJ, Jaspan T, McArthur DC, Walker DA. Effects of hydrocephalus after cerebellar tumor: a case-bycase approach. Pediatr Neurol 2011; 44(3): 193-201. http://dx.doi.org/10.1016/j.pediatrneurol.2010.09.010

[23] Massimino M, Gandola L, Barra S, Gianggaspero F, Casali C, Potepan $\mathrm{P}$, et al. Infant ependymoma in a 10-year AIEOP (Associazione Italiana Ematologia Oncologia Pediatrica) experience with omitted or deferred radiotherapy. Int J Radia Oncol Biol Phys 2011; 80(3): 807-814. http://dx.doi.org/10.1016/j.jirobp.2010.02.048

[24] Davis EE, Pitchford NJ, Jaspan T, McArthur D, Walker D. Development of cognitive and motor function following cerebellar tumour injury sustained in early childhood. Cortex 2010; 46(7): 919-932.

http://dx.doi.org/10.1016/j.cortex.2009.10.001
[25] Gerber NU, Zehnder D, Zuzak TJ, Poretti A, Boltshauser E, Grotzer MA. Outcome in children with brain tumours diagnosed in the first year of life: long-term complications and quality of life. Archiv Dis Child 2008; 93: 582-589.

http://dx.doi.org/10.1136/adc.2007.116202

[26] von Hoff K, Kieffer V, Habrand J, Kalifa C, Dellatolas G. Grill, $\mathrm{J}$. Impairment of intellectual functions after surgery and posterior fossa irradiation in children with ependymoma is related to age and neurologic complications. BMC Cancer 2008; 8: Article Number 15 http://dx.doi.org/10.1186/1471-2407-8-15

[27] Merchant TE, Kiehna EN, Li C, Xiong X, Mulhern RK. Radiation dosimetry predicts IQ after conformal radiation therapy in pediatric patients with localised ependymoma. Int $J$ Radia Oncol Biol Phys 2005; 63(5): 1546-1554. http://dx.doi.org/10.1016/j.ijrobp.2005.05.028

[28] Suc E, Kalifa C, Brauner R, Habrand JL, Terrier-Lacombe MJ, Vassal G, et al. Brain tumours under the age of three: The price of survival. Acta Neurochirurgica 1990; 106: 93-98. http://dx.doi.org/10.1007/BF01809448

[29] Merchant TE, Lee H, Zhu J, Xiong X, Wheeler G, Phipps S, et al. The effects of hydrocephalus on intelligence quotient in children with localized infratentorial ependymoma before and after focal radiation therapy. J Neurosurg (Pediatrics 2) 2004; 101(Suppl 2): 159-168.

[30] Van Veelen-Vincent MC, Pierre-Kahn A, Kalifa C, SainteRose C, Zerah M, Thorne J, et al. Ependymoma in childhood: prognostic factors, extent of surgery, and adjuvant therapy. $J$ Neurosurg 2002; 97: 827-835. http://dx.doi.org/10.3171/jns.2002.97.4.0827

[31] Di Pinto M, Conklin HM, Li C, Xiong X, Merchant TE. Investigating verbal and visual auditory learning after conformal radiation therapy for childhood ependymoma. Int $\mathrm{J}$ Radia Oncol Biol Phys 2010; 77(4): 1002-1008. http://dx.doi.org/10.1016/j.ijrobp.2009.06.003

[32] Conklin H, Li C, Xiong X, Ogg R, Merchant TE. Predicting change in academic abilities after conformal radiation therapy for localised ependymoma. J Clin Oncol 2008; 26(24): 39653970.

http://dx.doi.org/10.1200/JCO.2007.15.9970

[33] Kieffer-Renaux V, Bulteau C, Grill J, Levy-Piebois C, Couanet $D$, Pierre-Kahn A. Visual agnosia after treatment of a posterior fossa ependymoma in a 16-month-old girl. $J$ Child Neurol 2001; 16(9): 698-704.

http://dx.doi.org/10.1177/088307380101600917

[34] Merchant TE, Hua CH, Shukla H, Ying X, Nill S, Oelfke U. Proton versus photon radiotherapy for common pediatric brain tumors: Comparison of models of dose characteristics and their relationship to cognitive function. Pediatr Blood Cancer 2008; 51(1): 110-117.

http://dx.doi.org/10.1002/pbc. 21530

[35] Martinez-Climent J, Sanchez VC, Menor CE, Miralles AV, Tortajada JP. Scale for assessing quality of life of children survivors of cranial posterior fossa tumors. I Neuro-Oncol 1994; 22: 67-76.

http://dx.doi.org/10.1007/BF01058356

[36] Puget S, Boddaert N, Viguier D, Kieffer V, Bulteau C, Garnett $M$, et al. Injuries to inferior vermis and dentate nuclei predict poor neurological and neuropsychological outcome in children with malignant posterior fossa tumors. Cancer 2009; 115(6): 1338-1347. http://dx.doi.org/10.1002/cncr.24150

[37] Kieffer-Renaux V, Viguier D, Raquin M, Laurent-Vannier A, Habrand J, Dellatolas G, et al. Therapeutic schedules influence the pattern of intellectual decline after irradiation of posterior fossa tumors. Pediatr Blood Cancer 2005; 45: 814819. http://dx.doi.org/10.1002/pbc.20329

[38] Fouladi M, Langston J, Mulhern R, Jones D, Xiong X, Yang J, et al. Silent lacunar lesions detected by MRI of children with 
brain tumors: a late sequela of therapy. J Clin Oncol 2000; 18(4): 824-831.

[39] Poggi G, Liscio M, Galbiati S, Adduci A, Massimino M, Gandola L, et al. Brain tumors in children and adolescents: cognitive and psychological disorders at different ages. Psycho-Oncol 2005; 14: 386-395. http://dx.doi.org/10.1002/pon.855

[40] Seaver E, Geyer R, Sulzbacher S, Warner M, Batzel L, Milstein $\mathrm{J}$, et al. Psychosocial adjustment in long-term survivors of childhood medulloblastoma and ependymoma treated with craniospinal irradiation. Pediatr Neurosurg 1994; 20: $248-253$.

http://dx.doi.org/10.1159/000120798

[41] Sands SA, Oberg JA, Gardner SL, Whiteley JA, GladeBender JL, Finlay JL. Neuropsychological functioning of children treated Neuropsychological functioning of children treated with intensive chemotherapy followed by myeloablative consolidation chemotherapy and autologous hematopoietic cell rescue for newly diagnosed CNS tumors: an analysis of the Head Start II survivors. Pediatr Blood Cancer 2010; 54(3): 429-436. http://dx.doi.org/10.1002/pbc.22318

[42] Fouladi M, Gilger E, Kocak M, Wallace D, Buchanan G, Reeves C, et al. Intellectual and functional outcome of children 3 years old or younger who have CNS malignancies. J Clin Oncol 2005; 23(28): 7152-7160. http://dx.doi.org/10.1200/JCO.2005.01.214

[43] Law N, Bouffet E, Laughlin S, Laperriere N, Briere M, Strother D, McConnell D, et al. Cerebello-thalamo-cerebral connections in pediatric brain tumor patients: Impact on working memory. Neurolmage 2011; 56(4): 2238-2248. http://dx.doi.org/10.1016/j.neuroimage.2011.03.065

[44] Pulsifer MB, Delgado I, Tarbell N, Kuhlthau K, MacDonald SM, Yock TI. Neurocognitive outcomes after proton radiation for pediatric brain tumors. J Clin Oncol 2010; 28(15): Abstract No. 9508.

[45] Babikian T, Asarnow R. Neurocognitive outcomes and recovery after pediatric TBI: Meta-analytic review of the literature. Neuropsychol 2009; 23(3): 283-296.

http://dx.doi.org/10.1037/a0015268

[46] Mulder H, Pitchford NJ. Hagger MS, Marlow N. Development of executive function and attention in preterm children: $A$ systematic review. Develop Neuropsychol 2009; 34(4): 393421. http://dx.doi.org/10.1080/87565640902964524

[47] Riva D, Giorgi C. The cerebellum contributes to higher functions during development: Evidence from a series of children surgically treated for posterior fossa tumours. Brain 2000; 1051-1061.

http://dx.doi.org/10.1093/brain/123.5.1051

[48] Middleton FA, Strick PA. The cerebellum: an overview. Trends Neurosci 1998; 21(9): 367-369.

http://dx.doi.org/10.1016/S0166-2236(98)01330-7

[49] Ackerman $\mathrm{H}$, Hertich I. The contribution of the cerebellum to speech processing. J Neurolingust 2000; 13: 95-116. http://dx.doi.org/10.1016/S0911-6044(00)00006-3

[50] Lalonde R, Botez-Marquard T. Neuropsychological deficits of patients with chronic or acute cerebellar lesions. J Neurolinguist 2000; 13: 117-128.

http://dx.doi.org/10.1016/S0911-6044(00)00007-5

[51] Diamond A. Close interrelation of motor development and cognitive development and of the cerebellum and prefrontal cortex. Child Develop 2000; 71(1): 44-56. http://dx.doi.org/10.1111/1467-8624.00117

[52] Temple C. Developmental Cognitive Neuropsychology. Hove: Psychology Press 1997.

[53] Rose SA, Feldman JF. Memory and speed: Their role in the relation of infant information processing to later IQ. Child Develop 1997; 68(4): 630-641. http://dx.doi.org/10.2307/1132115

[54] Snowling, MJ. Language skills and learning to read. Psycholog 2004; 17(8): 438-441.

[55] Stanovich, KE. Matthew effects in reading: Some consequences of individual differences in the acquisition of literacy. Read Res Quart 1986; 21(4): 360-407. http://dx.doi.org/10.1598/RRQ.21.4.1

[56] Aarsen FK, Paquier PF, Reddingius RE, Streng IC, Arts WFM, Evera-Preesman M, et al. Functional outcome after lowgrade astrocytoma in childhood. Cancer 2006; 106(2): 396402.

http://dx.doi.org/10.1002/cncr.21612

\section{DOI: http://dx.doi.org/10.14205/2309-3021.2014.02.01.4}

(c) 2014 Morrall et al.; Licensee Pharma Publisher.

This is an open access article licensed under the terms of the Creative Commons Attribution Non-Commercial License (http://creativecommons.org/licenses/by-nc/3.0/) which permits unrestricted, non-commercial use, distribution and reproduction in any medium, provided the work is properly cited. 